\title{
Eurômos : rapport préliminaire sur les travaux réalisés en 2016
}

\author{
Abuzer Kızıl, Koray Konuk, Sönmez Alemdar, Laurent Capdetrey, Raymond \\ Descat, Didier Laroche, Enora Le Quéré, Francis Prost et Baptiste \\ Vergnaud
}

\section{(2) OpenEdition}

12 Journals

Édition électronique

URL : http://journals.openedition.org/anatoliaantiqua/463

DOI : 10.4000/anatoliaantiqua.463

Éditeur

IFEA

Édition imprimée

Date de publication : 1 mai 2017

Pagination : 161-185

ISBN : 978-2-36245-066-2

ISSN : $1018-1946$

\section{Référence électronique}

Abuzer Kızl, Koray Konuk, Sönmez Alemdar, Laurent Capdetrey, Raymond Descat, Didier Laroche, Enora Le Quéré, Francis Prost et Baptiste Vergnaud, «Eurômos : rapport préliminaire sur les travaux réalisés en 2016 », Anatolia Antiqua [En ligne], XXV | 2017, mis en ligne le 01 mai 2019, consulté le 19 décembre 2020. URL : http://journals.openedition.org/anatoliaantiqua/463 ; DOI : https://doi.org/ 10.4000/anatoliaantiqua.463 


\section{ANATOLIA ANTIQUA ESKI ANADOLU}

\section{XXV}

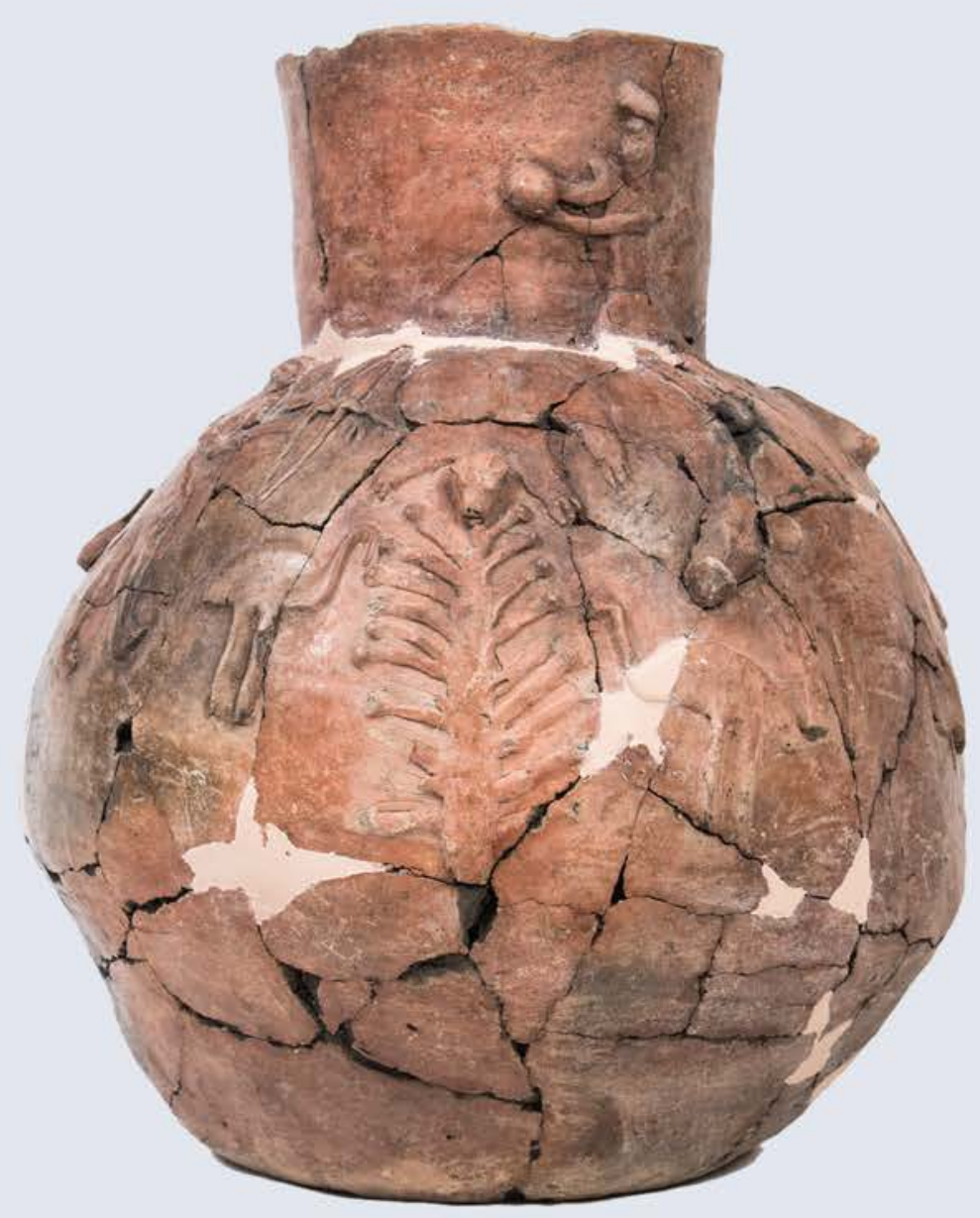

INSTITUT FRANÇAIS D'ETUDES ANATOLIENNES GEORGES-DUMEZIL

CNRS USR 3131

DE BOCCARD 


\section{TABLE DES MATIERES}

N. Pınar ÖZGÜNER et Geoffrey D. SUMMERS

The Çevre Kale Fortress and the outer enclosure on the Karacadağ at Yaraşl1

Abuzer KIZIL et Asil YAMAN

A group of transport amphorae from the territorium of Ceramus: Typological observations

Tülin TAN

The hellenistic tumulus of Eşenköy in NW Turkey

Emre TAŞTEMÜR

Glass pendants in Tekirdağ and Edirne Museums

Liviu Mihail IANCU

Self-mutilation, multiculturalism and hybridity. Herodotos on the Karians in Egypt (Hdt. 2.61.2) .....

\section{CHRONIQUES DES TRAVAUX ARCHEOLOGIQUES EN TURQUIE 2016}

Erhan BIÇAKÇI, Martin GODON et Ali Metin BÜYÜKKARAKAYA, Korhan ERTURAÇ, Catherine KUZUCUOĞLU, Yasin Gökhan ÇAKAN, Alice VINET

Les fouilles de Tepecik-Çiftlik et les activités du programme Melendiz préhistorique, campagne 2016

Çiğdem MANER

Preliminary report on the forth season of the Konya-Ereğli Survey (KEYAR) 2016

Sami PATACI et Ergün LAFLI

Field surveys in Ardahan in 2016

Erkan KONYAR, Bülent GENÇ, Can AVCI et Armağan TAN

The Van Tušpa Excavations 2015-2016

Martin SEYER, Alexandra DOLEA, Kathrin KUGLER, Helmut BRÜCKNER et Friederike STOCK The excavation at Limyra/Lycia 2016: Preliminary report

Abuzer KIZIL, Koray KONUK, Sönmez ALEMDAR, Laurent CAPDETREY, Raymond DESCAT, Didier LAROCHE, Enora LE QUERE, Francis PROST et Baptiste VERGNAUD

Eurômos : rapport préliminaire sur les travaux réalisés en 2016

O. HENRY et D. LÖWENBORG, Fr. MARCHAND-BEAULIEU, G. TUCKER, A. FREJMAN,

A. LAMESA, Chr. BOST, B. VERGNAUD, I. STOJANOVITC, N. CARLESS-UNWINN,

N. SCHIBILLE, Ö.D. ÇAKMAKLI, E. ANDERSSON

Labraunda 2016 


\section{CHRONIQUES DES TRAVAUX ARCHEOLOGIQUES EN TURQUIE \\ 2016}




\section{Abuzer KIZIL, Koray KONUK, Sönmez ALEMDAR, Laurent CAPDETREY, Raymond DESCAT, Didier LAROCHE, Enora LE QUERE, Francis PROST et Baptiste VERGNAUD*}

\section{EURÔMOS : RAPPORT PRELIMINAIRE SUR LES TRAVAUX REALISES EN 2016}

La deuxième campagne de la Mission archéologique française d'Eurômos du Ministère des Affaires étrangères et du Développement international eut lieu au mois d'août $2016^{1}$. Cette année a également vu la mise en place d'un projet de prospection accepté par la Direction des biens culturels et des musées et placé sous la direction d'Abuzer Kız1l. Les membres de l'Institut Ausonius mènent toutefois depuis l'été 2012 des travaux épigraphiques et numismatiques ainsi que des prospections à Eurômos et ses environs grâce à l'appui financier du Labex LaScArBx de l'université de Bordeaux et de l'Institut Ausonius (UMR 5607). Il convient aussi de mentionner les contributions de l'équipe "Mondes grecs archaïques et classiques" de l'UMR 7041 ArScAn (université Paris 1) et du GRHis, EA 3831 de l'université de Rouen, qui aident spécialement au volet archéologique de la mission. Eurômos est un site antique de Carie qui se situe à $12 \mathrm{~km}$ environ au Nord de Milas (ancienne Mylasa) au Nord-Ouest du petit village de Kızılcakuyu, au Sud-Est d'une plaine distincte de celle de Milas mais qui la prolonge. La situation d'Eurômos en fait la cité historique la plus importante au Nord de Mylasa jusqu'au littoral maritime antique qui ouvre sur Milet. Eurômos présente de ce fait une particularité étonnante car elle est la seule cité de Carie qui possède des vestiges substantiels qui n'ait jamais fait l'objet d'une étude spécifique, et qui ne dispose même d'aucun plan véritable, alors que tous les sites voisins comme Milet, Héraclée du Latmos, Labraunda, Amyzon, Alinda, Stratonicée, Mylasa, Iasos ont été ou sont étudiés. Une autre originalité d'Eurômos est de présenter les vestiges d'un temple, celui de Zeus Lepsynos, sans doute le mieux conservé de toute la Carie et qui a été régulièrement dessiné puis photographié depuis les premiers voyages savants au $18^{\mathrm{e}}$ siècle.

\section{L'AGORA}

(L. Costa, E. Le Quéré, Fr. Prost, J. Bernini, T. Lucas et J. Rivault)

Entre le 14 et le 28 août 2016, la deuxième campagne d'étude de l'agora d'Eurômos a pu être conduite par une équipe composée de Laurent Costa, ingénieur topographe du CNRS (ArScAn, UMR 7041), d'Enora Le Quéré (maître de conférences d'Histoire grecque, Université de Rouen, EA 3831 GRHis) et de Francis Prost (professeur d'Archéologie classique, Université Paris 1-Panthéon Sorbonne, ArScAn, UMR 7041). Elle a reçu le soutien de deux étudiantes de doctorat de l'Université Bordeaux Montaigne, Julie Bernini et Joy Rivault, ainsi que d'un étudiant de doctorat de l'Université Paris 1, Thierry Lucas.

Les objectifs de la campagne 2016 sur l'agora d'Eurômos ont été essentiellement des objectifs d'étude technique : il s'agissait 1) d'obtenir un plan topographique fiable de l'agora et de rassembler les données photographiques pour la mise en place d'une reconstitution en trois dimensions ; 2) d'engager l'inventaire systématique de tous les blocs actuellement disponibles sur le site pour la mise en œuvre de la reconstitution des portiques et de l'espace dans son ensemble ; 3 ) de poursuivre les réflexions et les observations sur la base des nouvelles données établies

*) La mission française d'Eurômos était composée en 2016 du chef de mission Koray Konuk, de Patrice Brun, Laurent Capdetrey, Raymond Descat, Baptiste Vergnaud ; de trois étudiants de doctorat : Julie Bernini, Joy Rivault, Olivier Vanwalleghem (Institut Ausonius, UMR 5607 et LaScArBx) ; Sönmez Alemdar (Université de Tübingen); Didier Laroche (ENSAS) ; Enora Le Quéré (GRHis, EA3831) ; Francis Prost, Laurent Costa ; d'un étudiant de doctorat : Thierry Lucas (ArScAn, UMR 7041) ; Baptiste Vergnaud (IFEA, Istanbul), travaillant sous l'autorité du directeur des fouilles Abuzer Kızıl (Université Sitkı Koçman de Muğla) et de la Direction générale des biens culturels et des musées du Ministère de la culture et du tourisme d'Ankara.

1) Nous avons également bénéficié cette année d'une aide financière de l'Institut Universitaire de France via Patrice Brun, membre senior de IUF. En outre, le Labex LaScArBx soutient un projet d'étude du système défensif d'Eurômos sous la direction de Patrice Brun et l'expertise de Baptiste Vergnaud. La mission archéologique française d'Eurômos est associée à l'équipe archéologique turque dirigée par Abuzer Kiz1l. 
depuis l'année dernière et des acquis, en particulier à propos du problème des ouvertures de l'agora.

\subsection{Fabrication du plan général de l'agora d'Eurômos}

L'objectif de la campagne conduite par L. Costa et Fr. Prost a été de produire un plan topographique du secteur de l'agora. En effet, nous n'avions pas de plan à disposition, sinon un plan faux levé sans moyen technique adéquat et non géoréférencé. Plusieurs technologies ont été mobilisées pour tenter de restituer une topographie aussi précise que possible de l'agora du site antique d'Eurômos.

Les éléments structurants de l'agora et les vestiges archéologiques en place ont d'abord été levés à l'aide d'un GPS cinématique temps réel de modèle R04 de marque Trimble. La Cinématique temps réel (Real Time Kinematic ou RTK) est une technique de positionnement par satellite basée sur l'utilisation de mesures issues des signaux émis par les systèmes GPS, GLONASS ou Galileo. Une première station de référence fournit des corrections en temps réel à une station mobile permettant d'atteindre une précision de l'ordre du centimètre. La précision nominale typique pour ces systèmes est de $1 \mathrm{~cm}$ horizontalement et $2 \mathrm{~cm}$ verticalement en moyenne.

A la suite de cette première collecte, dans l'optique de relever l'ensemble des blocs et de disposer ainsi d'un plan dit "pierre à pierre", une double campagne de photographies a été réalisée qui associe des images aériennes à très basse altitude produites à l'aide d'un drone DJI Phantom 3 piloté par K. Konuk et des photographies terrestres. Plus de 2000 images ont été ainsi collectées. Cette masse d'images permettra, après traitement, de modéliser l'état du site en 3D et de produire, sur la base de modèles photogrammétriques, une base de données géographique détaillée des vestiges de l'agora que nous pourrons compléter durant les campagnes suivantes. Cette même base constituera le socle pour la production de plans d'ensemble ou de secteurs, ainsi que pour les propositions de restitution en 3D.

\subsection{Inventaire des blocs du Portique Ouest de l'agora}

Nécessaire à la compréhension des édifices de l'agora en vue de leur restitution et indispensable pour une perception fine de leurs particularités, une campagne d'inventaire des blocs disponibles a été lancée par E. Le Quéré, assistée des trois étudiants.

Ce travail a pris la forme d'une base de données enregistrant systématiquement tous les blocs architecturaux de l'agora. Durant cette campagne 2016, c'est sur le Portique Ouest que se sont concentrés les efforts (Fig. 1). Ce portique est délimité au Nord et au Sud par deux piliers cordiformes qui assurent la jonction avec les Portiques Nord et Sud de l'agora. Long de $70 \mathrm{~m}$ (longueur interne, entre les piliers cordiformes), il présente un relativement bon état de conservation sur sa façade : nous disposons de la plupart des blocs de l'entablement, ainsi que de très nombreux fragments de colonnes, dont plusieurs en place, qui permettent de calculer l'entrecolonnement et de restituer ainsi 24 colonnes entre les deux piliers cordiformes. La hauteur totale des colonnes (sans chapiteau, H. $5 \mathrm{~m}$; avec le chapiteau, H. 5,34 m) avait déjà pu être établie ${ }^{2}$. L'emplacement du mur de fond ne peut en revanche être restitué avec assurance, ayant été complètement "mangé" par la construction de la route moderne quasi adjacente, mais la mise au jour de l'euthyntéria du mur de fond du Portique Sud suggère que l'articulation avec le Portique Ouest ne devait pas aller au-delà des $7 \mathrm{~m}$. Ce portique, affichant une façade d'ordre composite comme tous les autres portiques de l'agora, présente par ailleurs un degré de qualité et de finition des blocs que l'on n'observe nulle part ailleurs sur l'agora.

L'intégralité des blocs architecturaux visibles constituant le portique, pour la plupart effondrés sur place lors de la destruction de l'agora, a été répertoriée et inventoriée lors de cette campagne. Un premier relevé en plan du Portique Ouest effectué il y a quatre ans sous la direction d'A. Kizıl a servi de base de travail, en attendant de pouvoir se fonder sur le plan topographique entrepris cette année. Dixhuit secteurs ont été définis dans le portique (du Nord au Sud) afin de faciliter le travail d'inventaire et la numérotation. Chacun des blocs a donc été numéroté (numérotation continue allant de A200 à A496, portée à la fois sur la pierre et sur le plan), identifié, mesuré, décrit avec un maximum d'informations, dessiné sous forme de croquis et photographié. L'ensemble de ces informations ont été ensuite consignées dans une base de données réalisée à l'aide du logiciel File Maker Pro, comportant une fiche par bloc architectural et intégrant tous les dessins et photographies. Cette base permet ainsi une utilisation scientifique de toutes ces données, et 


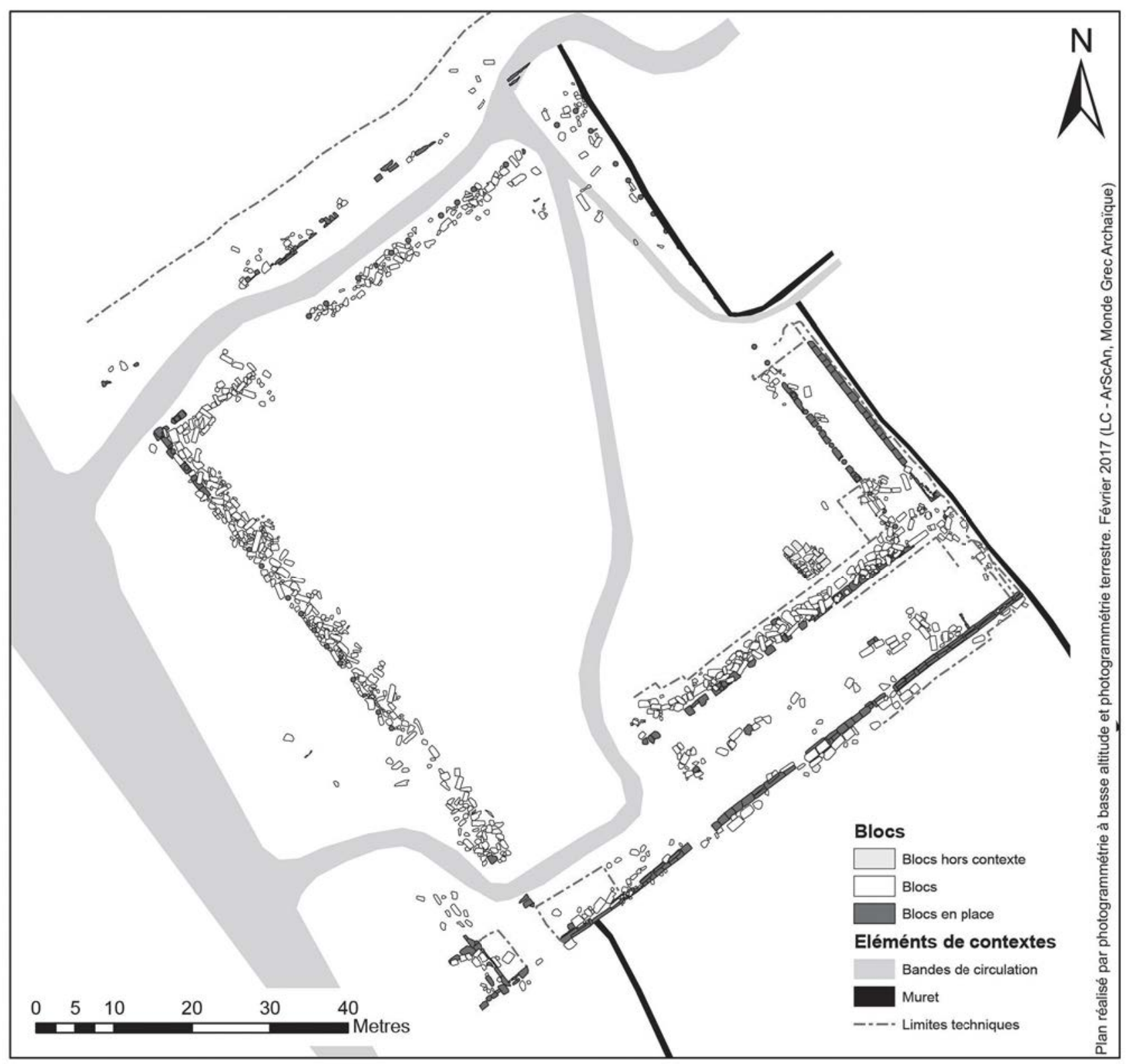

Fig. 1 : Relevé de l'agora.

devrait, à terme, considérablement faciliter la reconstitution architecturale des quatre portiques de l'agora.

Sur un total de 297 blocs architecturaux inventoriés pour le Portique Ouest, 200 ont pu, à ce stade de l'étude, être identifiés avec certitude : 9 blocs de stylobate, 49 fûts ou fragments de colonnes, 4 fûts de piliers cordiformes, 3 fragments d'un chapiteau cordiforme, 28 fragments ou blocs d'architrave dont 3 portant une inscription, 36 fragments ou blocs de frise, 59 fragments ou blocs de corniche, 7 fragments de parpaings et d'orthostates appartenant probablement au mur de fond du portique, et 5 fragments appartenant probablement à des bases ou petits monuments qui étaient élevés dans le portique (Fig. 2). Même si la plupart des blocs constituant le portique ont été dégagés, quelquesuns sont encore en partie enterrés, ce qui ne permet pas toujours de les identifier ni de les analyser en détail. Afin de parfaire l'étude architecturale de ces blocs, il conviendrait également de les déplacer et de les ranger, pour pouvoir les observer et les mesurer sur tous leurs angles. L'analyse devra bien évidemment être complétée si des fouilles venaient à être entreprises dans ce secteur de l'agora, car elles mettront probablement au jour de nouveaux blocs appartenant au portique. 


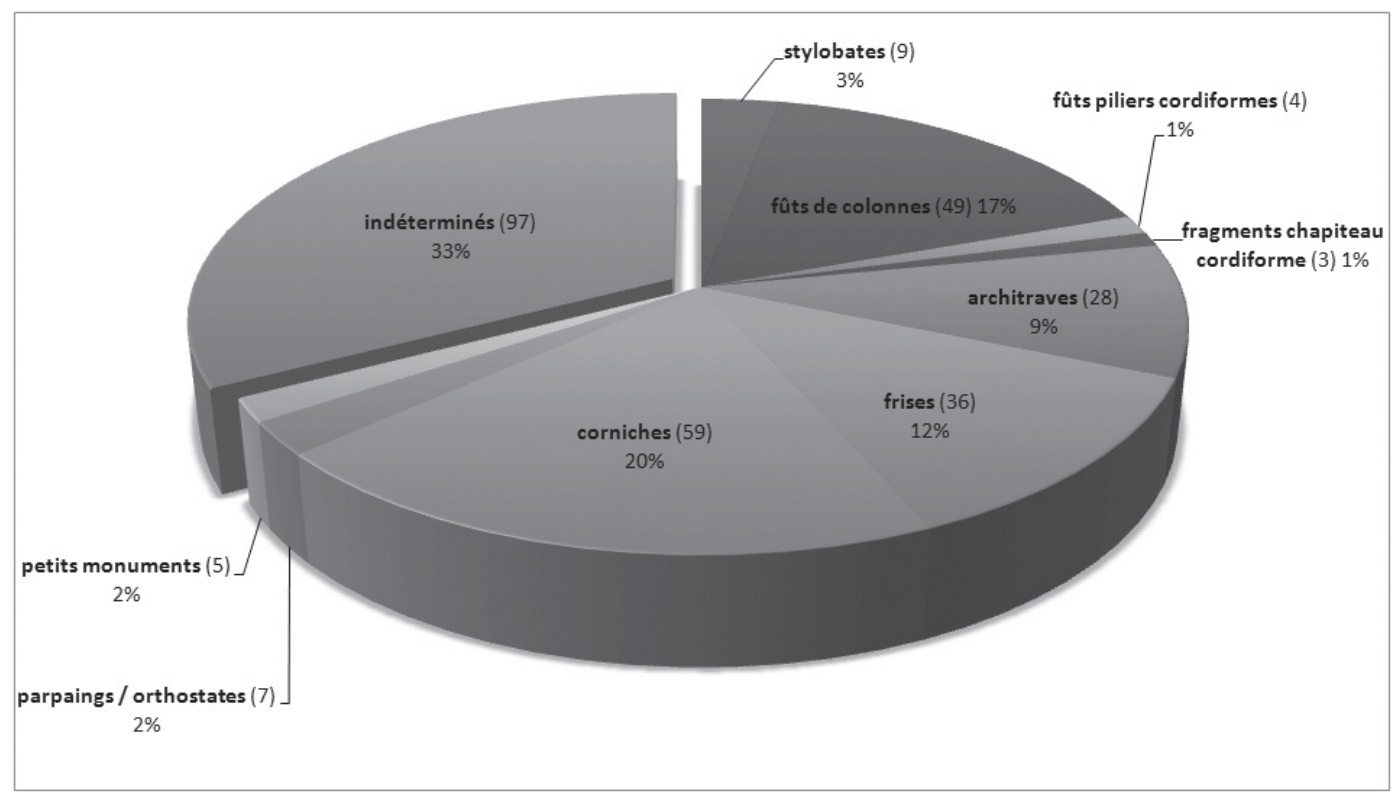

Fig. 2 : Identification des blocs architecturaux du Portique Ouest : répartition.

\subsection{Observations diverses}

La campagne de cette année a été encore l'occasion de poursuivre les observations architecturales des quatre portiques, en vue de leur reconstitution. Trois secteurs ont retenu tout spécialement l'attention: A) l'euthyntéria du mur de fond du Portique Sud et son angle avec le Portique Ouest ; B) les stylobates des quatre portiques de l'agora; C) enfin, l'ouverture entre le Portique Est et le Portique Sud.

A) Les nettoyages engagés par A. Kizil au Portique Sud, dans la prolongation des travaux effectués l'an passé, ont livré quelques trouvailles intéressantes pour la reconstitution et l'histoire de ce portique. Dans la partie orientale de la seconde nef, contre le mur du fond, un grand fragment de chapiteau ionique a été exhumé, très exactement entre le fût de colonne aux cannelures esquissées, et les orthostates. Cet emplacement indique que ce chapiteau devait à l'origine surmonter ce tambour aux cannelures engagées, mais inachevées. Nanti d'un abaque à kymation lesbique, orné d'une demi-palmette d'écoinçon ajourée seulement dans sa partie supérieure, doté d'une volute très régulière et d'un bouton central à peine bombé orné d'une fleur à quatre pétales très effacés (Fig. 3), ce chapiteau vient compléter les deux autres plus petits fragments, découverts l'an passé dans la même zone, et renforce les hypothèses chronologiques sur la date d'érection de cette colonnade centrale du Portique Sud.

En outre, à l'extérieur du mur de fond du Portique Sud, quasiment à la moitié de sa longueur, le

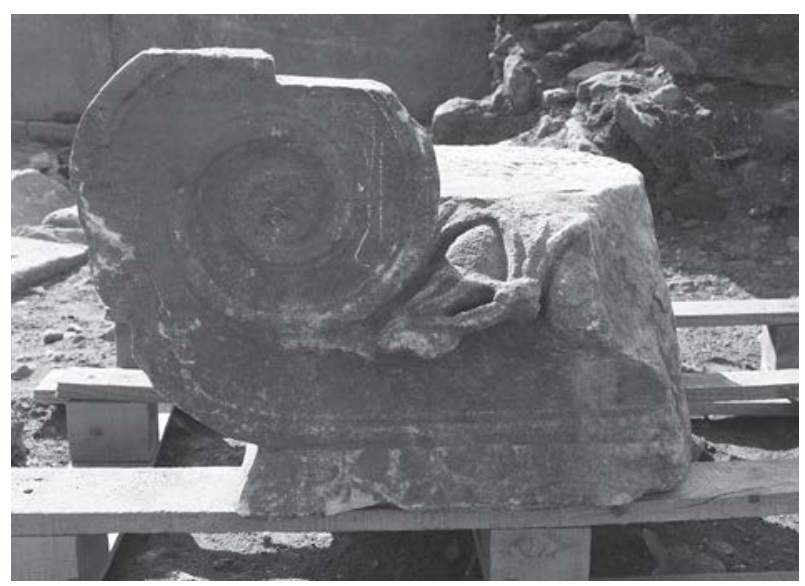

Fig. 3 : Chapiteau ionique.

nettoyage des abords a permis de mettre au jour un des grands blocs disposés à l'origine en parpaing sur les orthostates. Sur la tranche de ce bloc, une grande inscription conservée sur quatre lettres était profondément gravée, en lettres de $16 \mathrm{~cm}$ de hauteur. On lit - TOPO. La façon dont le bloc s'est effondré - à l'envers, juste devant les deux blocs d'orthostates couchées eux-mêmes à l'extérieur du portique, l'un sur l'autre - indique que l'inscription était disposée à l'intérieur du portique et courait presque à hauteur de poitrine, à 1,10 $\mathrm{m}-1,30 \mathrm{~m}$ au-dessus de l'euthyntéria.

Enfin, le nettoyage des blocs du Portique Ouest a permis aussi de dégager des fragments d'inscription : alors que deux fragments d'architrave avaient révélé 
l'an passé une inscription disposée sur les deux fasces de chacun des blocs, un troisième fragment, inscrit de la même manière, a été découvert cette année, et permet d'assurer la mention d'une stéphanéphorie.

B) Grâce encore aux nettoyages effectués par A. K1z1l, aux Portiques Sud et Est, nous disposons aujourd'hui de plusieurs informations nouvelles sur les stylobates des portiques. En effet, jusqu'à cette année, seuls les stylobates des Portiques Ouest et Nord étaient observables, sur une partie réduite à l'angle nord-ouest : les stylobates étaient constitués de grandes dalles strictement alignées sur le côté de l'agora et irrégulièrement taillées du côté de l'intérieur des portiques. Ces dalles n'étaient pas scellées les unes aux autres, mais jointives - à l'exception d'une dalle de réparation entre le pilier cordiforme nord-ouest et la première colonne du Portique Ouest, qui présente deux mortaises et qui, plus courte dans ses dimensions, a dû être complétée pour atteindre une surface comparable à ses voisines. $\mathrm{Au}$ Portique Sud, le dégagement du stylobate a permis de constater que non seulement il était constitué des mêmes grandes dalles, cette fois-ci strictement taillées et alignées sur le côté de l'agora comme sur le côté de l'intérieur du portique, mais aussi que les empreintes de la base des colonnes avaient été conservées assez précisément : les colonnes étaient scellées au stylobate par deux goujons latéraux, alors qu'au Portique Ouest, la base des colonnes n'était scellée que par un goujon central. Mais c'est encore une fois le Portique Est qui se singularise tout particulièrement. Le stylobate est constitué uniquement de dalles en remploi. Certaines d'entre elles présentent des mortaises qui n'ont pas leur pendant sur la dalle adjacente, ou encore l'une d'elle a conservé le souvenir d'une moulure tout juste démaigrie pour l'aplanissement de sa surface. Ces observations viennent confirmer le caractère distinct du Portique Est et sa chronologie plus récente que celle des trois autres portiques.

Ce n'est pas tout. Le dégagement du stylobate du Portique dorique Sud a permis de mettre au jour les empreintes des colonnes et, ainsi, de calculer l'entraxe. Il faut constater qu'il est de 2,80 m environ, alors qu'il n'est que de 2,70 m environ au Portique Nord et au Portique Ouest. Cet entraxe plus fort au Sud conduit à ne restituer, du coup, que 24 colonnes sur la façade sud (sans compter la colonne d'angle cordiforme), alors qu'on peut restituer 25 colonnes au Nord (sans compter la colonne d'angle cordiforme). La raison de cette différence est probablement due au fait qu'il fallait prévoir un nombre de colonnes ioniques de la colonnade intérieure qui puissent répondre aux exigences suivantes : une colonne ionique toutes les deux colonnes doriques, et une colonne ionique en face de la cordiforme et une autre en face de la dernière colonne dorique avant l'articulation avec le Portique Est. Il fallait donc au Sud 25 colonnes doriques $(24+$ la cordiforme), et non 26 ( 25 + la cordiforme) comme c'était le cas au Nord. Le dégagement des dalles de fondations des colonnes ioniques, cinq actuellement repérables, méritera d'être repris et précisé l'an prochain.

C) Enfin, grâce aux nettoyages de l'an passé engagés à l'angle méridional du Portique Est, nous avons pu procéder cette année à plusieurs observations sur la zone qui a certainement été l'une des entrées de l'agora (Fig. 4). La dernière colonne du Portique Sud est située dans l'axe des colonnes du Portique Est, mais entre cette colonne et la première de la colonnade est, l'entrecolonnement est de $2,40 \mathrm{~m}$ environ, et non de 2,01 à 2,03, comme constaté ailleurs. On retrouve cet entrecolonnement exceptionnel à l'angle nord-est, entre la dernière colonne du Portique Nord et la première du Portique Est, sans qu'on puisse le vérifier avec précision puisque la zone n'est pas encore complètement dégagée. Cet écartement plus fort dans ces deux endroits est dû au fait qu'à l'Ouest sont disposés aux angles les deux piliers cordiformes, dont les demi colonnes engagées ajoutent bien quelque $40 \mathrm{~cm}$ à l'entrecolonnement. Or ces $2,40 \mathrm{~m}$ se retrouvent exactement dans le fond du Portique Est, entre le mur de fond qui fait très exactement retour en face de la première colonne du Portique Est, et un bloc exhumé situé dans l'axe de la colonnade du Portique Sud, vestige sans doute d'un mur permettant de négocier le retour du Portique Sud. C'est à cet endroit qu'ont été dégagés l'an passé les blocs d'arche, dont nous avons pu calculer le rayon cette année : il est de 1,20 m. Il convient donc de le restituer au-dessus de ce passage.

La moisson d'informations de la campagne 2016 a donc été très fructueuse. L'an prochain, il sera nécessaire de poursuivre l'inventaire et les observations architecturales, mais il sera plus nécessaire encore d'engager des sondages pour apporter des réponses précises à de nombreuses questions actuellement laissées en suspens : l'articulation entre le Portique Est et le Portique Nord, le système d'ouverture hypothétique au Nord du Portique Est, ou encore l'emplacement du mur de fond du Portique Ouest. 


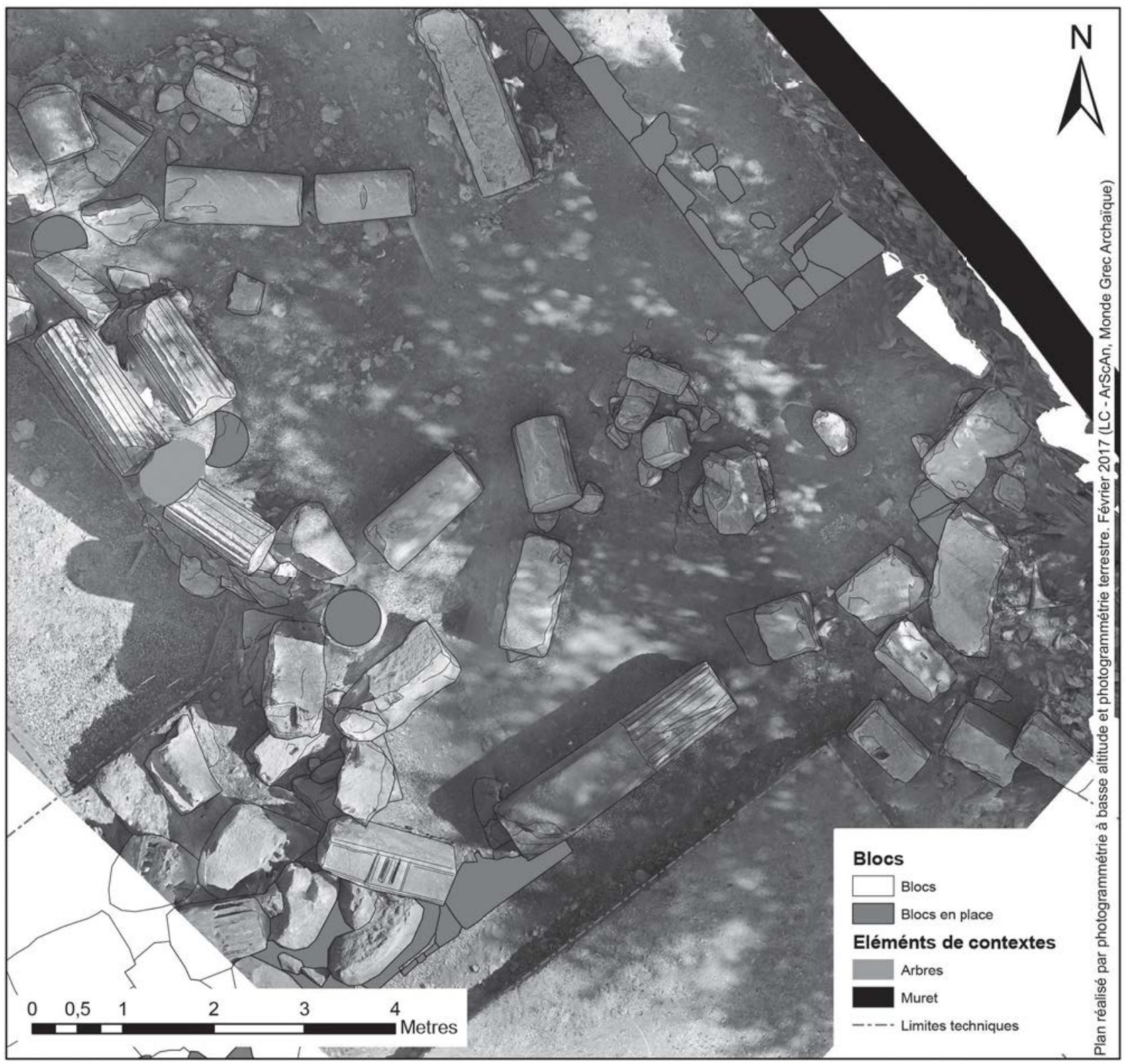

Fig. 4 : Planimétrie de l'angle sud-est de l'agora.

\section{LA MURAILLE}

(B. Vergnaud)

La campagne de l'année 2016 a porté sur la partie nord de la muraille (Fig. 5). En 2015, la tour hexagonale située au sommet d'une petite colline au point le plus septentrional du site avait été en partie étudiée (Fig. 6) ${ }^{3}$. Il était donc approprié de poursuivre les recherches dans ce même secteur défendu par deux tours supplémentaires : une tour quadrangulaire et une tour ronde. Le choix de se pencher plus avant sur ce secteur du rempart permet de poursuivre les objectifs que nous nous étions fixés : 1) dater la construction et les possibles réfections de la muraille, 2) étudier les tours en vue d'établir une typologie et 3) localiser les portes de la cité, dessiner leur plan et envisager leur relation avec la trame urbaine de la cité.

L'ensemble des chercheurs qui se sont intéressés à la muraille d'Eurômos s'accordent pour dater sa construction à l'époque hellénistique ${ }^{4}$. Cette datation reposant essentiellement sur des critères architecturaux

3) Vergnaud 2016b : 325-333.

4) Ibid. : n. 5. 
et historiques, le recours à des fouilles archéologiques s'avérait indispensable. En 2014, un sondage d'ampleur limitée aux abords d'une tour carrée située à proximité du temple nous avait permis de constater que le secteur avait été réoccupé à une époque tardive, après l'abandon du système défensif. Les fouilles de 2015 avaient porté sur la tour hexagonale située au Nord du circuit. Aucune couche contemporaine de la muraille n'avait survécu à son démantèlement mais le type de la tour et le matériel découvert en relation avec les fondations nous ont permis d'envisager une datation préliminaire au tournant des $3^{\mathrm{e}}-2^{\mathrm{e}} \mathrm{s}$. av. J.-C. en attendant une étude plus avancée du matériel ${ }^{5}$.

Les travaux de cette année se sont focalisés sur la tour carrée et la tour ronde situées toutes deux en contrebas de la tour hexagonale (Fig. 5 et 7). Un sondage d'ampleur limitée a été réalisé dans la première dans l'espoir de mettre au jour des couches d'occupation et de prélever du matériel associé aux fondations, l'objectif étant de dater la construction de la tour. Une autre tranchée a été réalisée contre le parement extérieur du mur est de la tour car nous supposions que cette tour défendait une des portes de la cité. Il s'agissait donc de voir si la tour était associée ou non à un mur de courtine. Aucune fouille en profondeur n'a été réalisée sur la tour ronde mais nous avons procédé à un nettoyage soigneux de la surface des murs afin de mettre son plan en évidence et de comprendre sa relation avec les courtines adjacentes ${ }^{6}$.

\subsection{La tour quadrangulaire (Fig. 8)}

La tour mesure environ $7 \mathrm{~m} \times 8 \mathrm{~m}$ de côté. Ses murs sont à double parement, construits en appareil pseudo-isodome à carreaux et boutisses d'une épaisseur de 1,50 m en moyenne (Fig. 9-10). Son élévation conservée est de 3 assises au maximum. Il est très probable que la tour fut chaînée au mur de fortification mais un mur de pierres sèches moderne recouvre le mur en question et la jonction avec la tour sur la partie ouest. Nous avons cherché à savoir si la tour était connectée à la muraille ou si elle protégeait l'un des accès à la cité par le biais d'une tranchée
(Fig. 10). Celle-ci n'a pas permis d'obtenir de réponse très claire à ce sujet. Aucune trace d'un mur de courtine n'a été découverte mais ce secteur est très perturbé. L'angle sud-est a été complètement arraché et à environ deux mètres vers l'Est un canal a été creusé à l'époque moderne, oblitérant les possibles traces de l'enceinte. L'hypothèse de l'existence d'une porte dans ce secteur reste donc ouverte mais demeure très vraisemblable du fait de la topographie du terrain.

L'étude de la tour carrée a été facilitée par le fait qu'elle n'était pas totalement recouverte par la végétation, à l'exception d'un olivier qui a poussé dans le remplissage du mur à l'angle nord-est de la tour. Après un nettoyage de la surface des murs et de l'espace à l'intérieur de la tour, nous avons procédé à un sondage à l'angle nord-ouest de la tour. Celui-ci ne nous a pas permis de mettre en évidence une couche d'occupation contemporaine de l'utilisation du bâtiment. Nous sommes rapidement parvenus à une couche de réoccupation tardive (Fig. 11). Celle-ci est composée d'une surface de circulation argileuse très compacte, le remplissage de laquelle contenait des tessons de céramique antique et tardive, notamment un pithos fragmentaire en place. Le fond de ce contenant est manquant. Sous cette couche située à la limite des fondations nous sommes parvenus à la couche de remplissage liée aux fondations et donc à la construction de la tour (Fig. 12). Cette couche a livré du matériel qui devrait nous permettre d'apporter des éléments de datation sur la construction de la tour ${ }^{7}$. Le médiocre état de conservation de cette tour n'a pas permis de parvenir à une connaissance précise du bâtiment. Aucune couche contemporaine de l'occupation de la tour n'a été conservée et il nous est donc impossible de savoir si le premier niveau de l'édifice a pu servir de lieu de repos pour les gardes comme nous avons pu le démontrer à Labraunda ${ }^{8}$.

\subsection{La tour ronde (Fig. 13-20)}

La seconde partie des travaux de terrain s'est focalisée sur la tour ronde située entre la tour carrée et la tour hexagonale. L'ouvrage est très mal conservé :

5) Toutes les datations sont av. J.-C. sauf indications contraires.

6) Les travaux de cette année n'auraient pu être menés efficacement sans le concours de deux valeureux archéologues, Thierry Lucas (doctorant, Université Paris I) et Olivier Vanwalleghem (doctorant, Université Bordeaux Montaigne), que je remercie chaleureusement. Mes remerciements vont également à Laurent Costa pour le travail de topographie qu'il a réalisé ainsi qu'à Koray Konuk pour les prises de vues par drone. Je remercie également Abuzer Kız1l, le directeur de la mission, pour son aide ainsi que l'ensemble de l'équipe d'Eurômos.

7) Une étude précise de ce matériel n'a pu être réalisée. Le matériel de cette tour et de la tour hexagonale fera l'objet d'un examen lors de la prochaine campagne et les résultats seront publiés dans le prochain rapport.

8) Voir notamment la Tour 2 de la forteresse de l'acropole (Vergnaud 2016a : 406-407). 


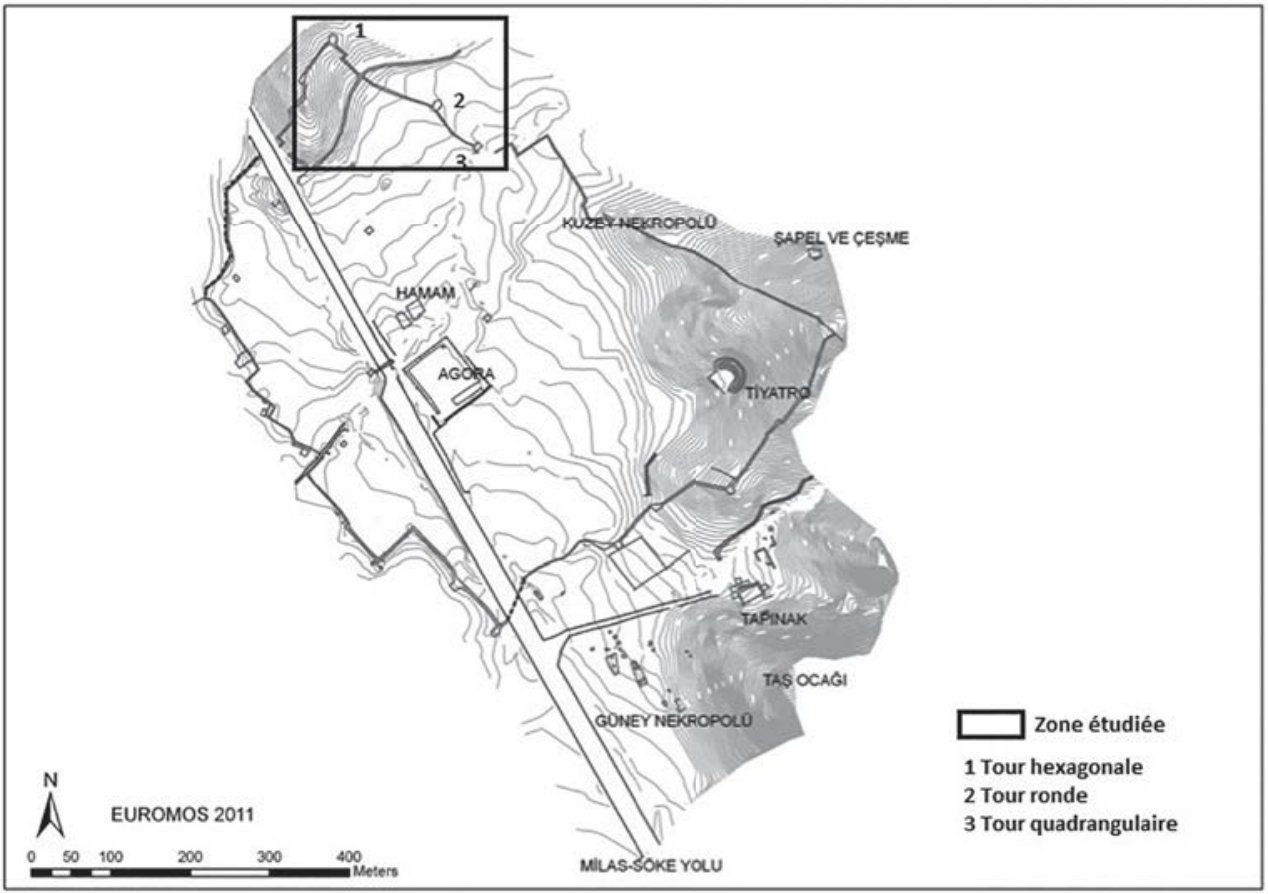

Fig. 5 : Plan de l'enceinte (mission archéologique d'Eurômos).

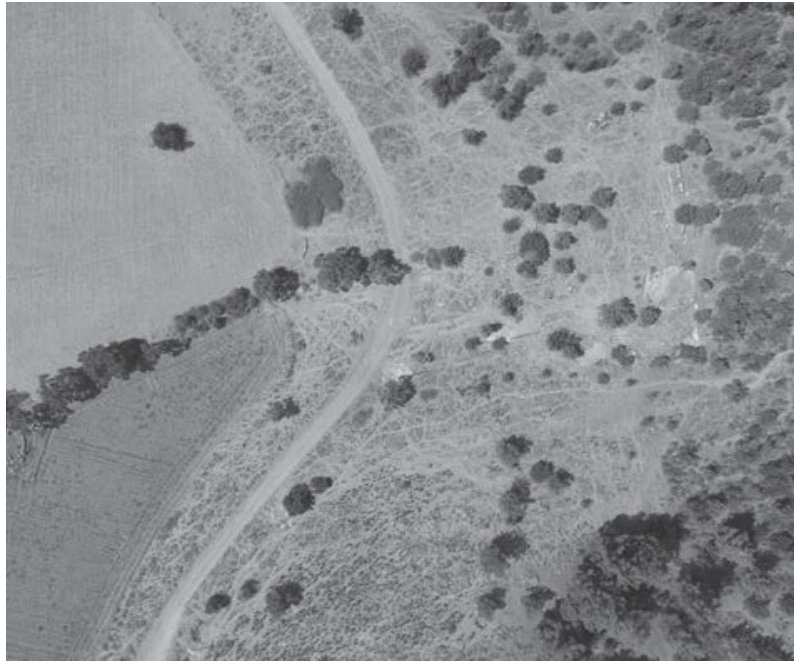

Fig. 6 : Section nord de la muraille. Tour hexagonale (droite) et ronde (gauche)

(K. Konuk).

seule la surface d'une assise était visible à notre arrivée. Le plan de la tour était relativement lisible mais il nous a fallu procéder à un nettoyage d'ensemble de la surface des murs pour le connaître en détail. La tour forme un arrondi très régulier percé d'un couloir d'accès. Ses murs sont composés d'un mur à double parement construits en carreaux et boutisses

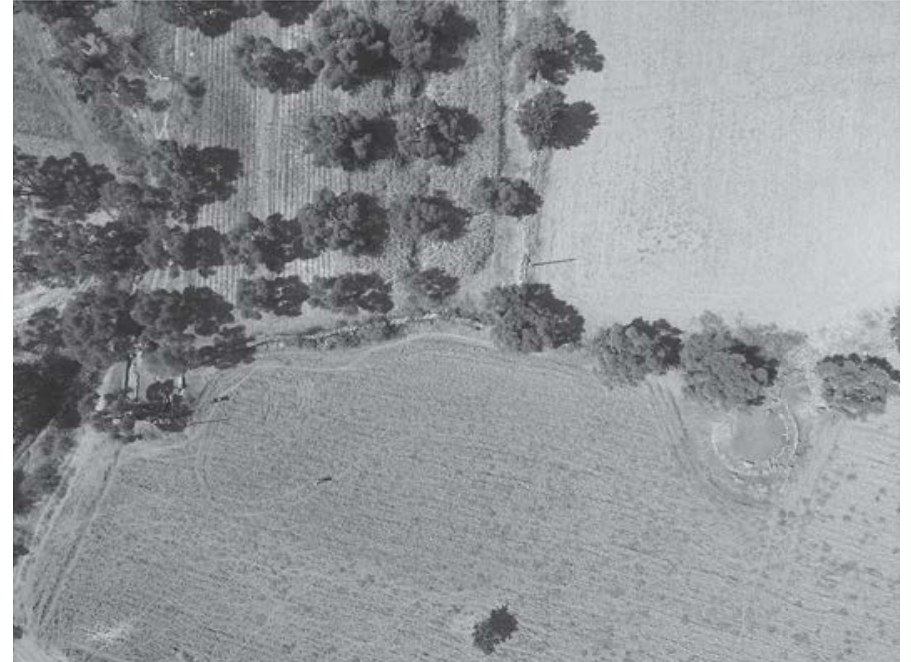

Fig. 7 : Section nord de la muraille. Tour ronde (droite) et quadrangulaire (gauche)

(K. Konuk).

(Fig. 14-15). L'alternance semble assez régulière sur la parement extérieur de la tour : un carreau pour une boutisse. Elle n'est pas régulière sur le parement intérieur : on remarque notamment une succession de 6 boutisses dans le mur ouest (Fig. 14 et 16). Les blocs sont plus massifs sur le parement extérieur. Le diamètre de la tour est très légèrement 


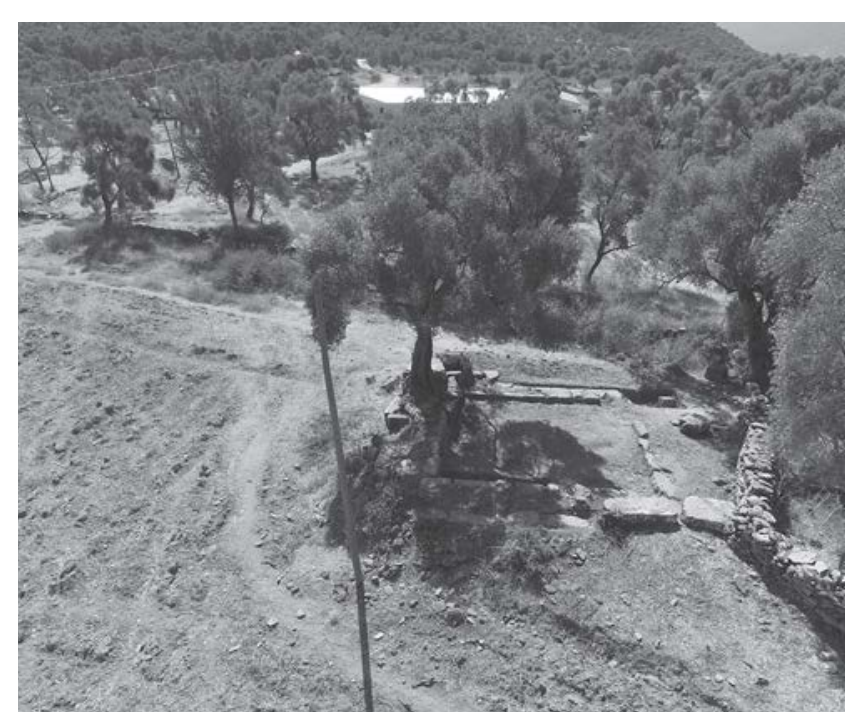

Fig. 8 : Tour quadrangulaire. Vue vers le Sud (K. Konuk).

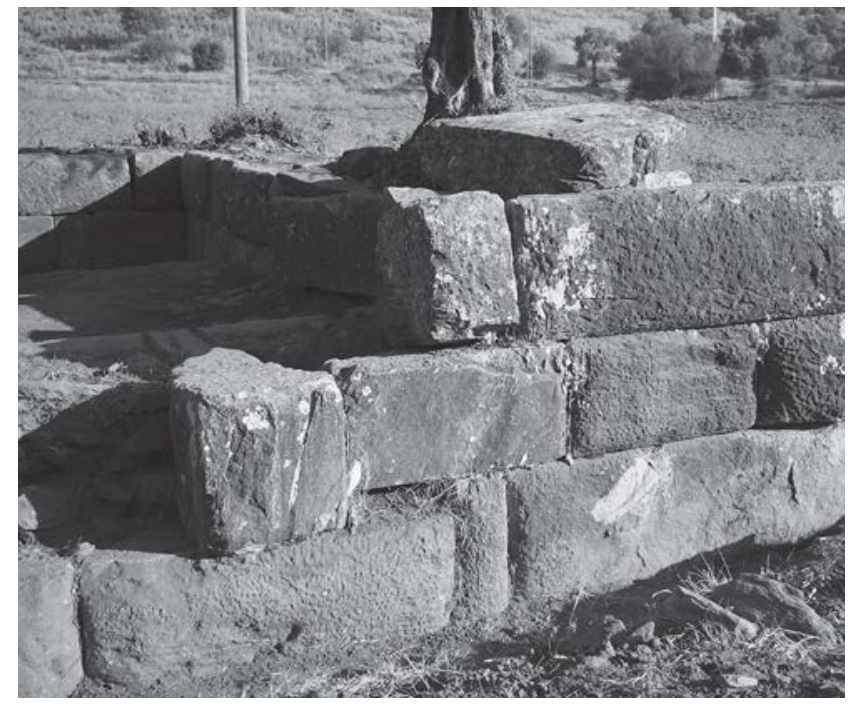

Fig. 9 : Tour quadrangulaire. Appareil. Vue vers le Nord (B. Vergnaud).

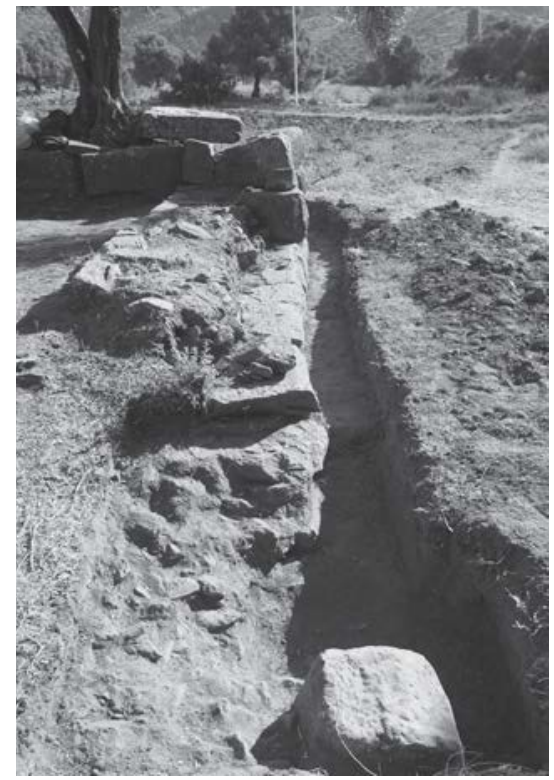

Fig. 10 : Tour quadrangulaire. Tranchée le long du parement extérieur (B. Vergnaud).

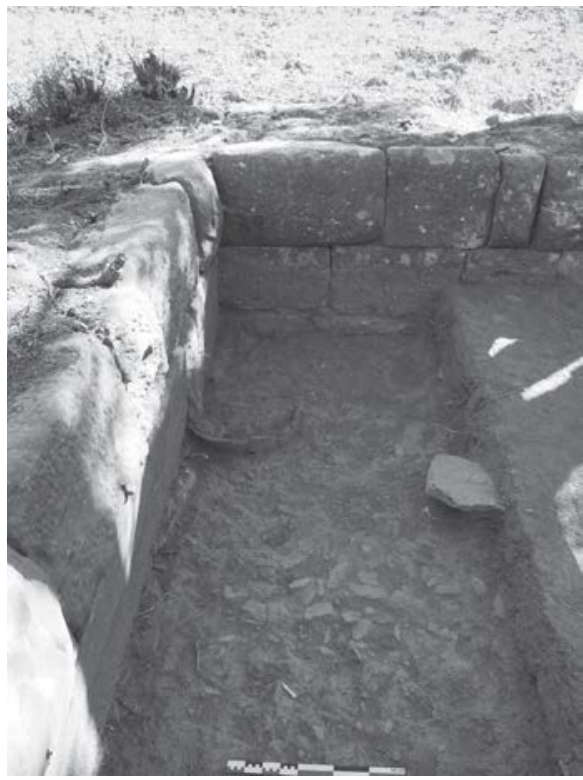

Fig. 11 : Tour quadrangulaire. Sondage dans l'angle intérieur de la tour. Couche tardive avec pithos (B. Vergnaud).

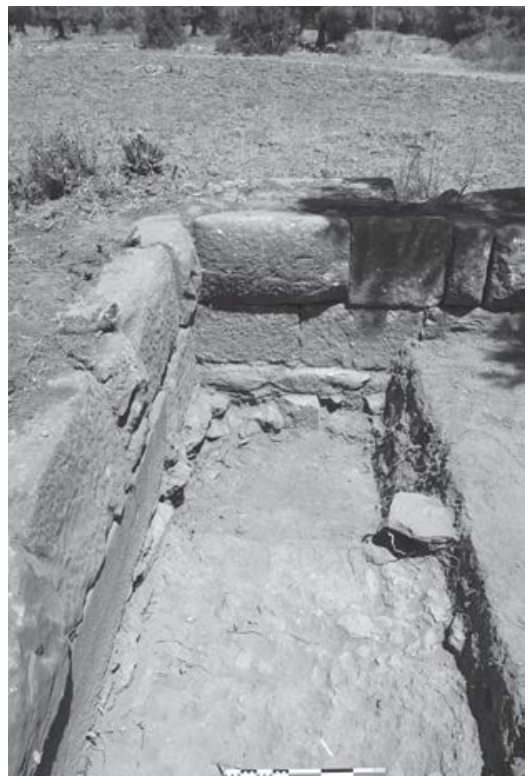

Fig : 12. Tour quadrangulaire. Sondage dans l'angle intérieur de la tour. Fondations

(B. Vergnaud). supérieur à $10 \mathrm{~m}$ d'un parement extérieur à l'autre9. Les murs oscillent entre 1,45 $\mathrm{m}$ et 1,60 m d'épaisseur. Le parement intérieur dans la partie est n'est pas visible. Les blocs ont en partie été arrachés et recouverts par une construction tardive composée de tuiles et de briques.
La connexion entre la tour et le mur de fortification n'a pas pu être mise en lumière. Un mur en pierre sèche recouvre en effet la muraille et la partie arrière de la tour. Le nettoyage du sommet des murs de la tour a révélé certains éléments inattendus qui permettent, sans avoir eu recours à une véritable fouille

9) Les tours de la porte d'Eétioneia au Pirée sont d'un diamètre similaire (10 et $11 \mathrm{~m})$. Celles de Pergè présentent un diamètre de $11,50 \mathrm{~m}$ (Hellman $2010: 333)$ et celle de Sidè, un diamètre légèrement supérieur à $10 \mathrm{~m}$ (McNicoll 1997: 145). 


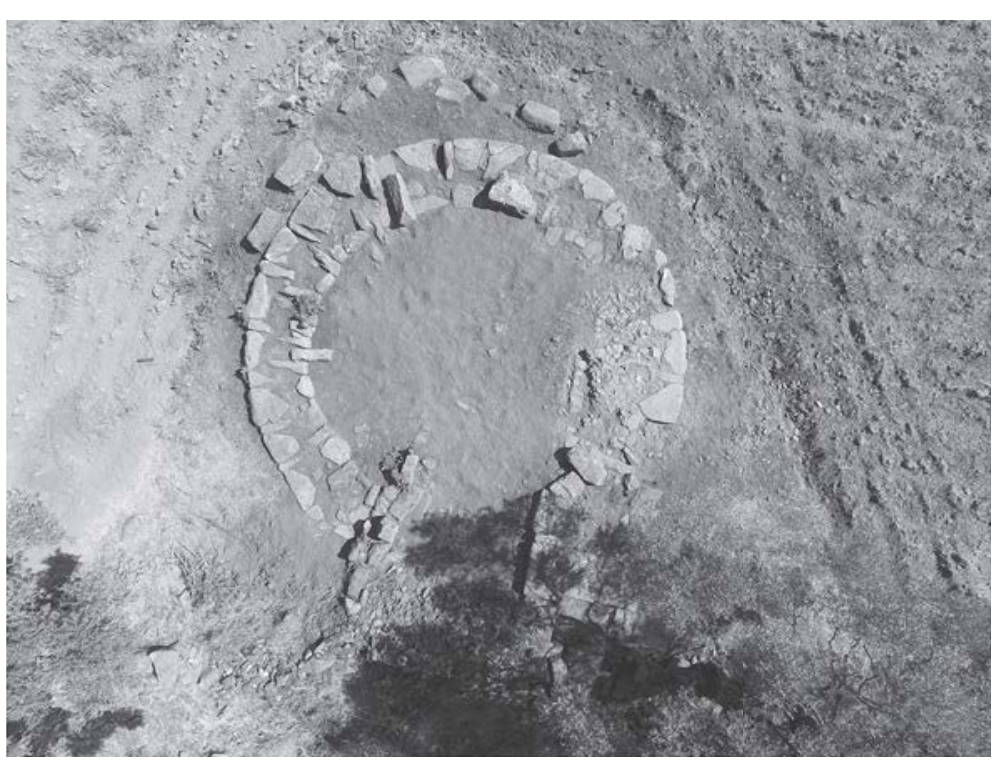

Fig : 13. Tour ronde. Vue aérienne (K. Konuk).

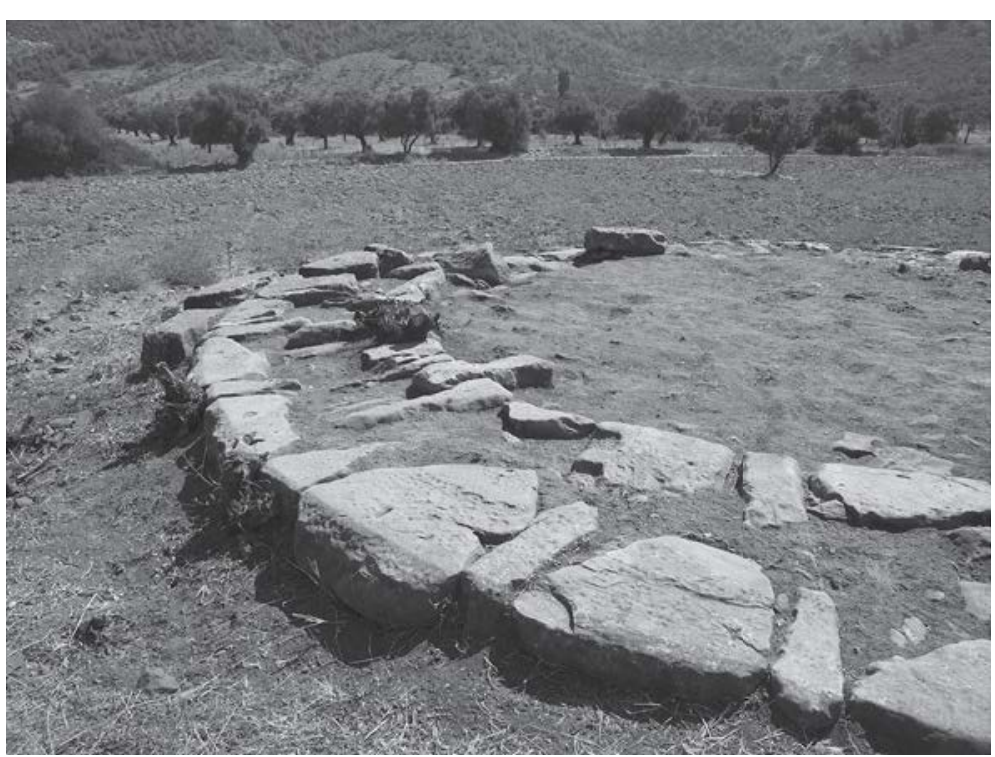

Fig : 15. Tour ronde. Mur ouest. Appareil (B. Vergnaud).

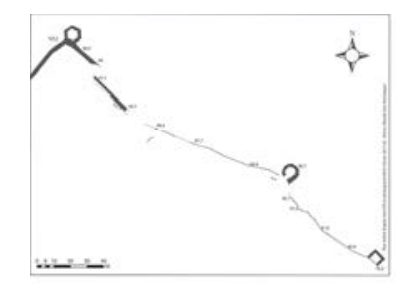

Eurômos 2016

Tour circulaire sur la

section nord-ouest du rempart
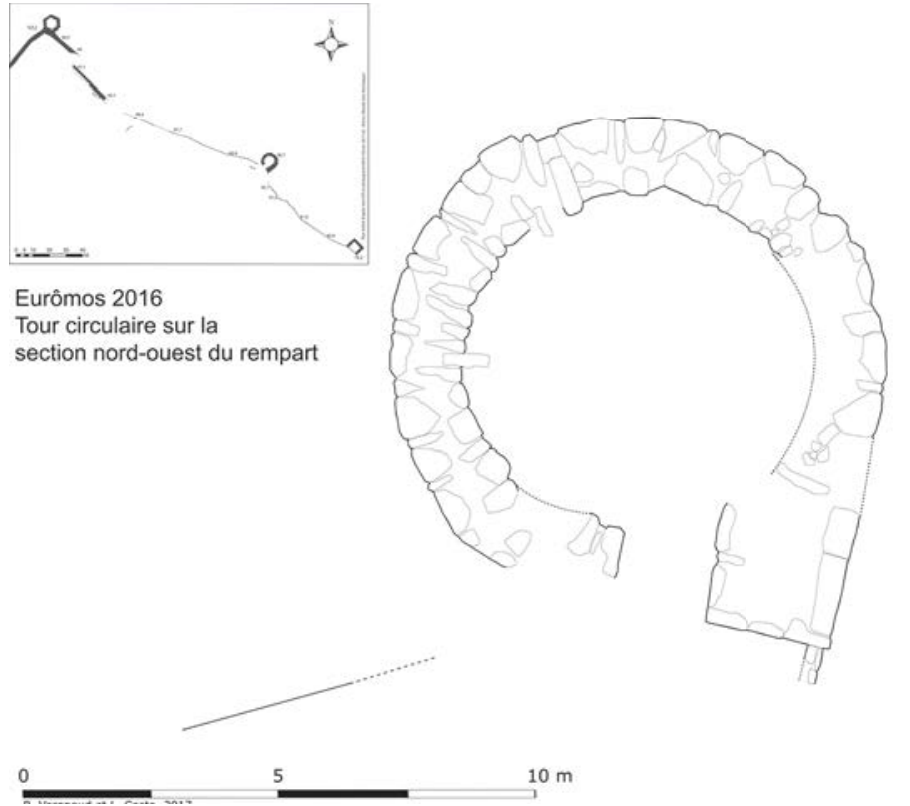

Fig. : 14. Tour ronde. Plan provisoire (B. Vergnaud).

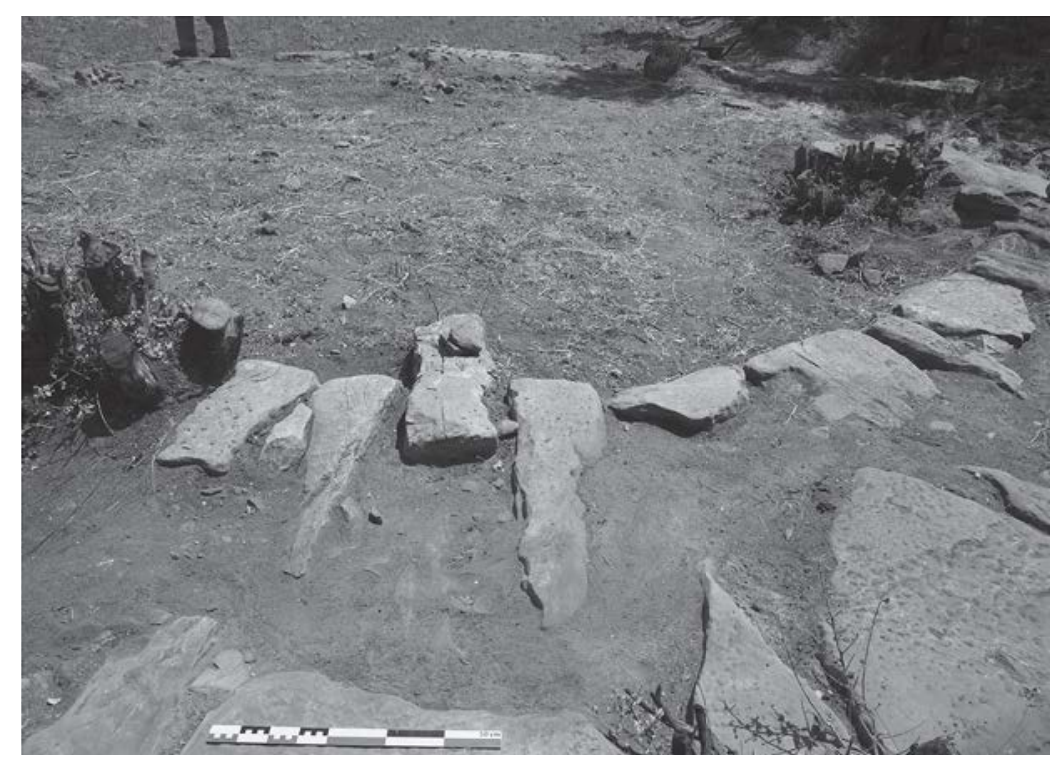

Fig : 16. Tour ronde. Mur ouest. Boutisses (B. Vergnaud). 
en profondeur, de suggérer que cette tour pourrait être un ajout au système de fortification original ou a pu subir des opérations de réfection. En premier lieu, nous avons pu observer la présence d'un bloc en remploi dans le parement intérieur sur la partie ouest de la tour (Fig. 17). Ce bloc, utilisé comme un carreau, présente des entailles destinées à recevoir des poutres. Il appartient à un bâtiment plus ancien, peut-être à la superstructure d'une tour antérieure à la tour ronde. Cette idée est appuyée par deux éléments supplémentaires. Le premier correspond à l'existence d'un massif rectangulaire situé à l'angle sud-est de la tour (Fig. 18). Les murs sont parfaitement rectilignes et ne décrivent aucun arrondi comme celui observable de l'autre côté du couloir d'entrée. Le second indice correspond à la présence d'un petit mur à double cours qui s'appuie contre l'angle de ce massif rectangulaire et qui devait sans doute faire la liaison avec la courtine (Fig. 14 et 19). L'absence de chaînage avec le mur de la tour et la faible épaisseur de ce mur laissent penser qu'il fut ajouté a posteriori pour lier la tour à la courtine, peut-être pour combler l'espace entre ces derniers. De l'autre côté du couloir d'entrée, le mur que l'on remarque en surface est une addition postérieure, sans doute très tardive et n'est pas en relation avec la tour ronde $^{10}$. Il est constitué de remplois. Enfin, lors du dégagement du sommet du mur est, en avant du massif rectangulaire, nous avons découvert une construction composée de briques et de tuiles contenues par deux petits murs se rencontrant à angle droit (Fig. 20 et 13). Il est possible qu'une partie des tuiles aient appartenues à la couverture de la tour. La fonction et la date exacte de cet aménagement restent inconnues ${ }^{11}$.

Plusieurs indices suggèrent que le secteur a été remanié et que la muraille a fait l'objet d'une réfection. Il nous faut donc envisager l'hypothèse selon laquelle la tour pourrait être un ajout au système de défense original. On a pu chercher à renforcer cette partie des défenses, peut-être du fait de la présence d'une porte à proximité de ce secteur ou simplement parce que cette partie de la muraille se trouvait en terrain plat et était donc vulnérable à une éventuelle attaque. La tour offrait une défense panoramique et offrait une meilleure défense dans cette configuration topographique ${ }^{12}$. L'idée selon laquelle la tour ronde s'est appuyée contre ou a été construite sur une tour précédente, quadrangulaire, mérite d'être envisagée car de telles transformations existent sur d'autres sites et notamment à Halieis où trois des six tours rondes avaient des prédécesseurs rectangulaires ${ }^{13}$.

\subsection{Conclusion et pistes chronologiques}

Au terme de la troisième campagne de recherche à Eurômos, beaucoup de questions restent en suspens et il nous est toujours impossible de proposer une datation très précise pour la construction de la muraille. Cependant, il nous est permis de suggérer que cette dernière a vraisemblablement subi un réaménagement partiel. La tour ronde située dans la partie nord du périmètre fortifié ne semble pas avoir fait partie du projet de construction initial comme l'indiquent l'utilisation d'un bloc en remploi et la connexion inhabituelle avec le mur de courtine. En ce qui concerne la tour carrée, on ne sait si elle appartenait à une porte mais cela paraît vraisemblable. La présence d'une porte dans ce secteur expliquerait d'ailleurs la construction de la tour ronde puisque ce type de tour est souvent utilisé pour défendre des angles d'un rempart ou des portes ${ }^{14}$. $\mathrm{Si}$ aucune interruption du périmètre fortifié n'est visible entre la tour carrée et la tour ronde ni entre cette dernière et la tour hexagonale, il est possible qu'une telle tour défendait l'approche d'une ouverture dans la muraille même de manière indirecte. L'étude de la céramique mise au jour dans la tour hexagonale en 2015 et dans la tour quadrangulaire en 2016 devrait nous permettre de préciser la datation du mur de fortification et de ces différentes composantes. Dans l'attente de ces résultats, la datation repose essentiellement sur des critères typologiques et techniques.

Les indications chronologiques rassemblées au cours des trois dernières campagnes laissent penser que la muraille d'Eurômos pourrait avoir été construite à partir de la fin du $3^{\mathrm{e}} \mathrm{s}$. Une telle datation est favorisée par plusieurs indices. D'abord, il existe une grande proximité entre le traitement des blocs

10) Ce mur ne figure pas sur le plan ci-joint.

11) Puisqu'il n'appartient pas à la tour, qu'il est visiblement tardif, nous avons choisi de ne pas le fouiller et de le recouvrir pour assurer sa protection.

12) Le terme de "défense panoramique" est emprunté à J.-P. Adam 1982 : 62.

13) McAllister $2005: 33$.

14) Winter $1971: 193$. 


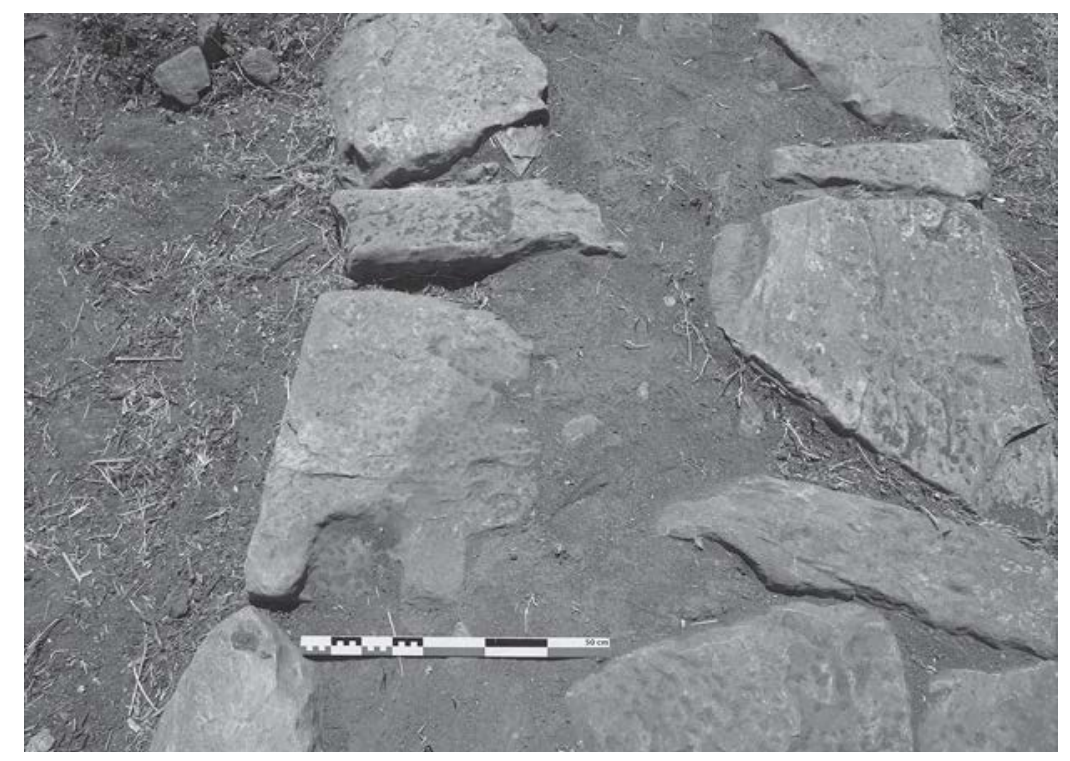

Fig. 17 : Tour ronde. Mur ouest. Bloc en remploi (B. Vergnaud).

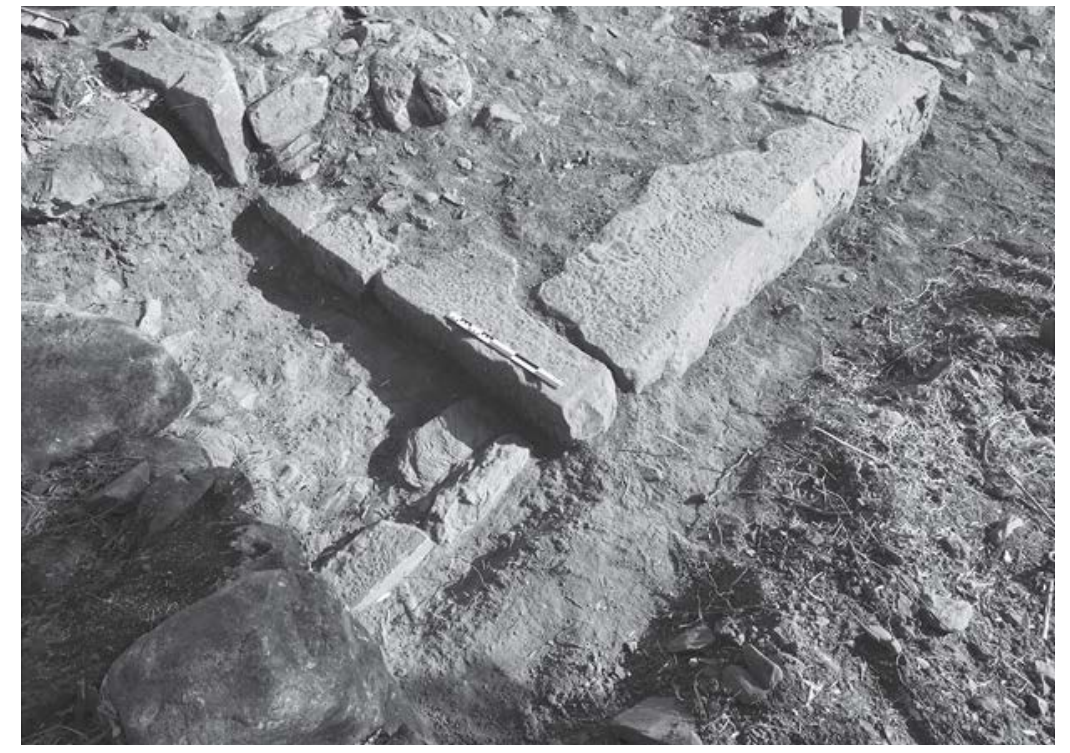

Fig. 19 : Tour ronde. Mur joignant la tour à la courtine (B. Vergnaud).

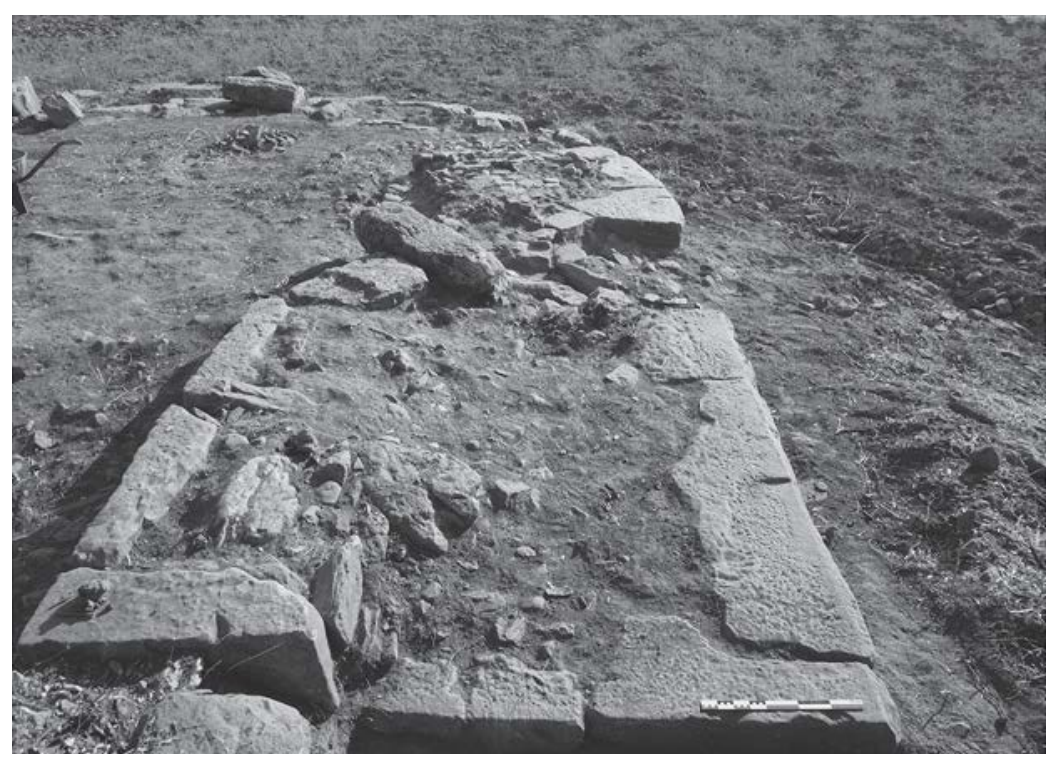

Fig. 18 : Tour ronde. Mur est. Massif rectangulaire (B. Vergnaud).

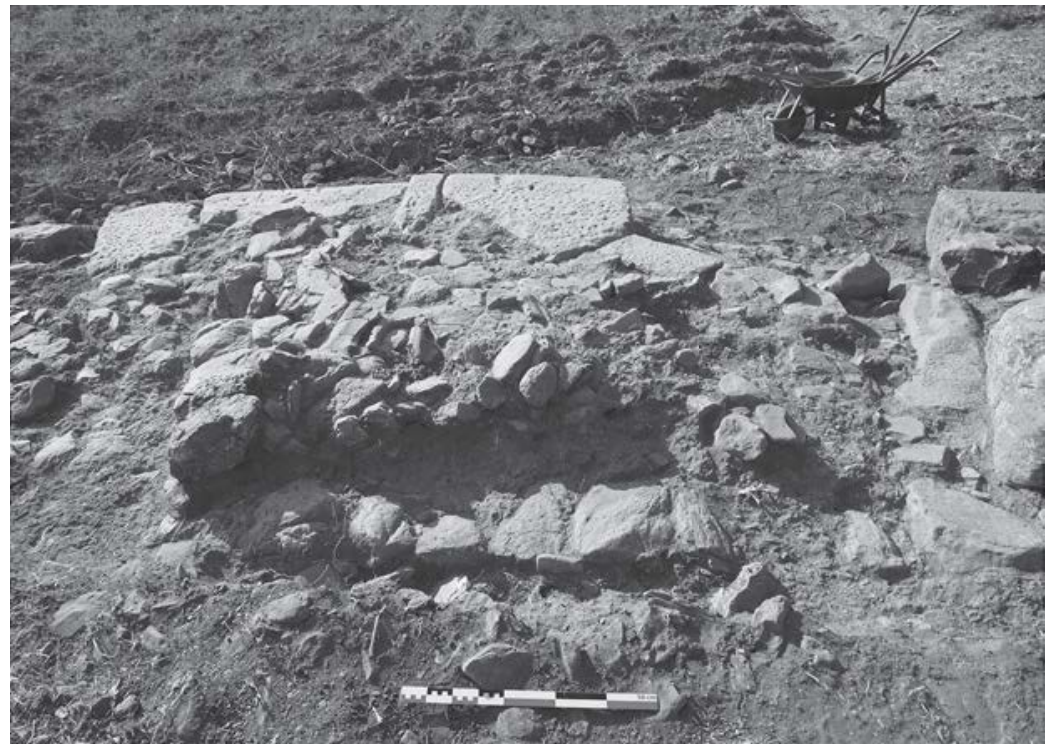

Fig. 20 : Tour ronde. Mur est. Construction tardive non identifiée (B. Vergnaud).$$
\text { non identifiée (B. Vergnaud). }
$$ 
de la tour carrée sud et ceux de l'ekklesiasterion de Priène daté de vers $200^{15}$. Ensuite, les fouilles de la tour hexagonale ont livré des tuiles de types très similaires à ceux découverts dans les contextes archéologiques de la forteresse de Labraunda et datés du tournant des $3^{\mathrm{e}}-2^{\mathrm{e}} \mathrm{s} .{ }^{16}$ La tour elle-même par son plan n'est pas sans rappeler des ouvrages similaires du Sud-Ouest de l'Asie Mineure. Des tours pentagonales et hexagonales ont été identifiées à Labraunda, Hyllarima, Harpasa, Samos, Milet ou encore Oinoanda ${ }^{17}$. Toutes ces tours sont datées entre la fin du $4^{\mathrm{e}} \mathrm{s}$. et le $1^{\mathrm{er}} \mathrm{s}$. sur des bases architecturales ou historiques. Cependant, l'une d'entre elles - la tour pentagonale de Labraunda - a fait l'objet d'une fouille stratigraphique et a pu être datée sur des bases matérielles fiables et ce à partir du dernier quart du $3^{\mathrm{e}} \mathrm{s}{ }^{18}$. D'un point de vue architectural, les tours de Labraunda et d'Eurômos partagent peu de points communs. Néanmoins, l'apparition d'un tel type de construction - une tour d'artillerie polygonale - en Carie intérieure et à proximité d'Eurômos, fournit une piste chronologique intéressante. En ce qui concerne les tours rondes, les datations ne sont pas mieux assurées. M.-C. Hellmann suggère que ces dernières furent "très appréciées en Asie Mineure à partir des années 200", bien qu'elles soient présentes en Carie, notamment à Alabanda, dès $350^{19}$. La datation des tours d'Oinoanda, d'Iasos (tours semi-circulaires), de Cnide, et de Pergè entre la fin du $3^{\mathrm{e}} \mathrm{s}$. et le $1^{\mathrm{er}} \mathrm{s}$. vont dans le sens de cette affirmation ${ }^{20}$. Si les tours rondes existent depuis bien avant l'époque classique, il semble tout de même qu'il y ait une recrudescence des tours circulaires, en fer à cheval ou à façade incurvée à l'époque hellénistique tardive ${ }^{21}$. La présence de plusieurs tours rondes à Eurômos et le fait qu'elles soient associées à au moins une tour polygonale est un indice supplémentaire pour envisager une datation au tournant des $3^{\mathrm{e}}-2^{\mathrm{e}} \mathrm{s}$. Il s'agit en tout cas d'une piste chronologique qu'il faut poursuivre en parallèle à une étude stratigraphique des tours de la cité.

\section{LE TEMPLE DE ZEUS LEPSYNOS ET LES THERMES DE l'AGORA}

(D. Laroche et S. Alemdar)

Les axes de travail, à propos du temple de Zeus, ont été les suivants :

- Mise en œuvre d'un catalogue général des blocs errants.

- Vérifications sur les vestiges en place afin de finaliser le plan restitué du temple.

- Etude sur les éléments constitutifs de l'élévation.

- Couverture photographique du temple à l'aide d'un drone (K. Konuk) afin de réaliser un modèle 3D de la ruine dans son état actuel (logiciel Photoscan).

- Travaux divers (autel).

Nous avons profité d'échanges, sur le terrain, avec Taylan Doğan, qui prépare une thèse sur cet édifice sous la direction de Abuzer Kızıl. Une jeune architecte, Pelda Kaya, a participé à certains relevés.

\subsection{TEMPLE DE ZEUS LEPSYNOS}

\subsubsection{Le catalogage général des blocs}

Le catalogage général des blocs est une étape indispensable pour la connaissance de l'architecture du temple. Cette année, les blocs ont été numérotés à partir de 100 jusqu'à 800 , ce qui couvre les $2 / 3$ environs des blocs non en place gisant autour de la ruine. Seuls les fragments ne présentant pas de faces travaillées n'ont pas été numérotés. Le catalogue est réalisé sur FileMaker et comportera, pour chaque bloc, outre sa description par champs de recherche, un dessin et une photo (Fig. 21). Outre la reconnaissance de la provenance des blocs, l'inventaire permettra d'évaluer le niveau de conservation des différentes parties de l'ouvrage et, partant, les possibilités de reconstruction.

15) Vergnaud 2016b : 333 avec références.

16) Ibid. : 331 .

17) Labraunda : Vergnaud 2014 : 107-122 ; 2015 : 316-330 ; 2016a : 397-412 ; Hyllarima : Brun 1994 : $193-204$; Harpasa : Debord et Varinlioğlu $2011: 29-43$; Samos : Kienast 1978 : 85-95 ; Milet : von Gerkan 1935 : 49-52, Oinoanda : McNicoll 1997 : 120 126. La liste n'est pas exhaustive.

18) Vergnaud 2016a: 398-403.

19) Hellmann $2010: 332$. Notons toutefois que les exemples sont rares et pas nécessairement datables de vers 200.

20) Oinoanda : fin 3e-début $2^{\mathrm{e}}$ s. (McNicoll $1997: 120-126$; 155-156), Iasos-mur continental (Pimouguet-Pédarros 2000 : 348), Cnide : $3^{\mathrm{e}}-2^{\mathrm{e}}$ s. (McNicoll $1997: 58-59$, n. 78) ; Pergè : seconde moitié du I ${ }^{\mathrm{er}} \mathrm{s}$. (Martini $2016: 220-231$ ). La liste n'est pas exhaustive.

21) Voir notamment McNicoll $1997:$ 7, 104. 


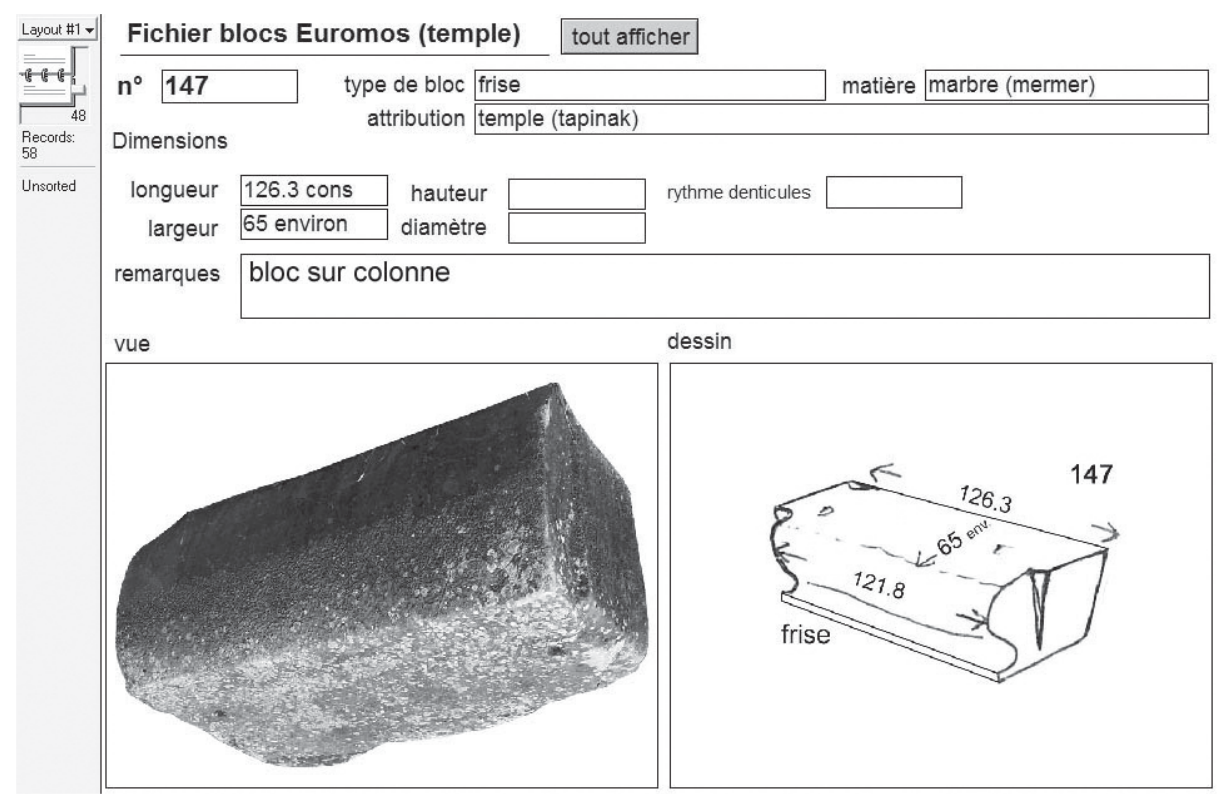

Fig. 21 : Exemple de fiche du catalogue général des blocs.

Dans cette optique, nous avons eu la surprise de constater l'absence complète de blocs provenant du fronton ouest, alors que le fronton semble complet. Plutôt que d'expliquer cette absence par une disparition ultérieure, contradictoire avec l'état de la ruine de la façade occidentale, il nous paraît plus logique de conclure à un inachèvement de l'édifice à l'Ouest. Cette constatation, inattendue, est moins surprenante lorsqu'on songe aux nombreux édifices inachevés de la région : temples de Claros et Didymes, mausolées de Bélévi et de Milas, etc. Elle nous oblige à poser la question de l'existence d'une toiture, dont aucun élément, pour l'instant, n'a été retrouvé.

Enfin, ce catalogage permettra de gérer la réorganisation des dépôts, dans le but de dégager des espaces de travail pour une future intervention sur l'édifice et le regroupement par type de bloc de l'ensemble des pièces errantes. Ce dernier objectif est indispensable pour rechercher les recollages de pièces brisées.

Nous avons procédé, à cette occasion, à plusieurs essais de modélisation en 3 dimensions de blocs errants. Ces essais (Fig. 22) permettent de préciser les conditions de prise de vue des blocs (accessibilité des faces, éclairage) pour un résultat exploitable.

\subsubsection{Restitution du temple}

Un certain nombre d'observations sont venues confirmer ou compléter celles faites l'an dernier, au niveau du plan du temple (Fig. 23) :
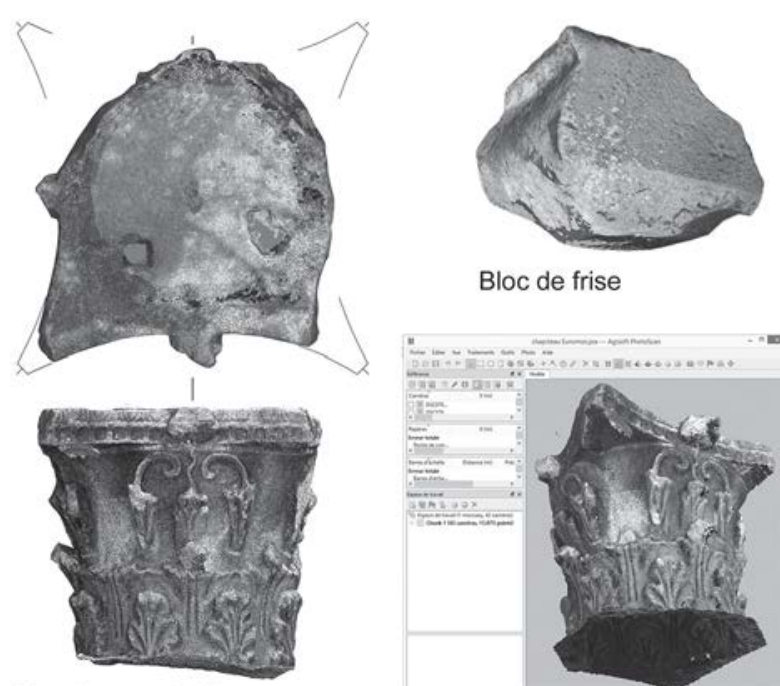

Bloc de frise

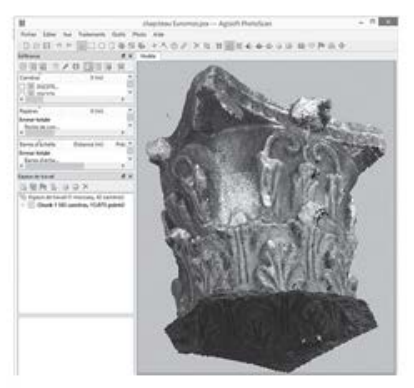

Chapiteau corinthien

Fig. 22 : Exemple de modélisation de blocs du temple avec le programme Photoscan.

- La restitution de quatre colonnes en avant du prodomos, qui ne peut se faire à partir du dallage reconstruit récemment, est assurée par l'existence d'encastrement pour une grille dans le prolongement des antes latérales de la cella.

- Sur la banquette nord de la cella se trouvait un baldaquin dont les traces de pose sont encore visibles (Fig. 24). Deux fragments de colonnettes provenant de ce baldaquin ont été identifiés. Il y a de fortes probabilités pour que ce baldaquin ait accueilli une statue cultuelle, en complément de celle qui se 


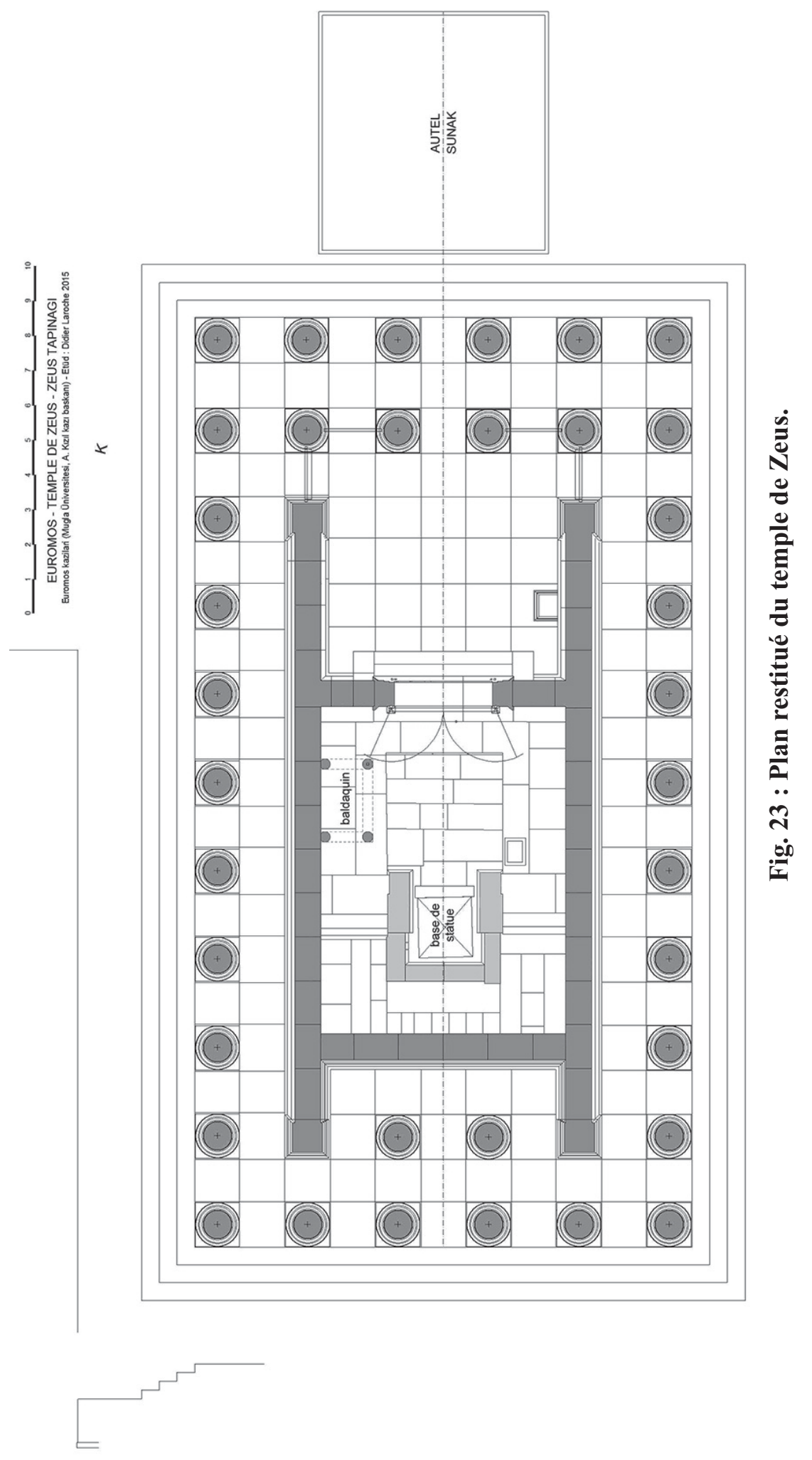




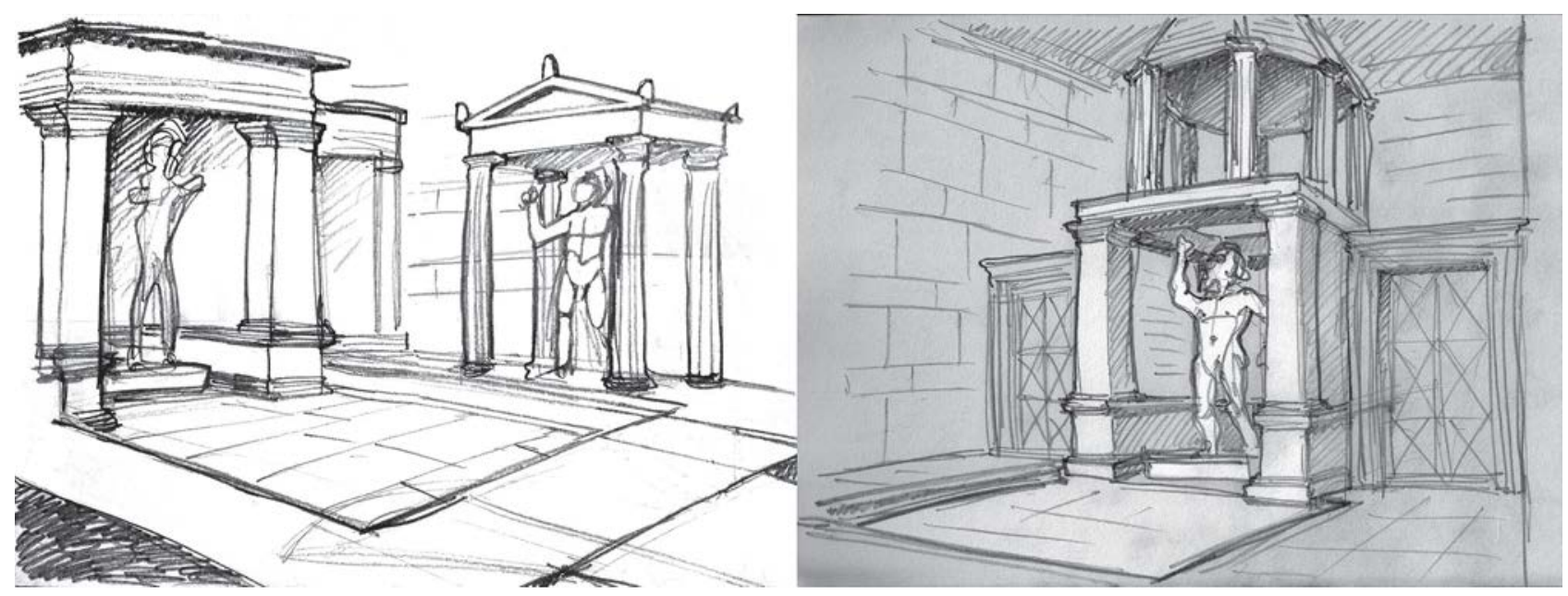

Fig. 24 : Essais de restitution du naiskos et du baldaquin pour statues de la cella.
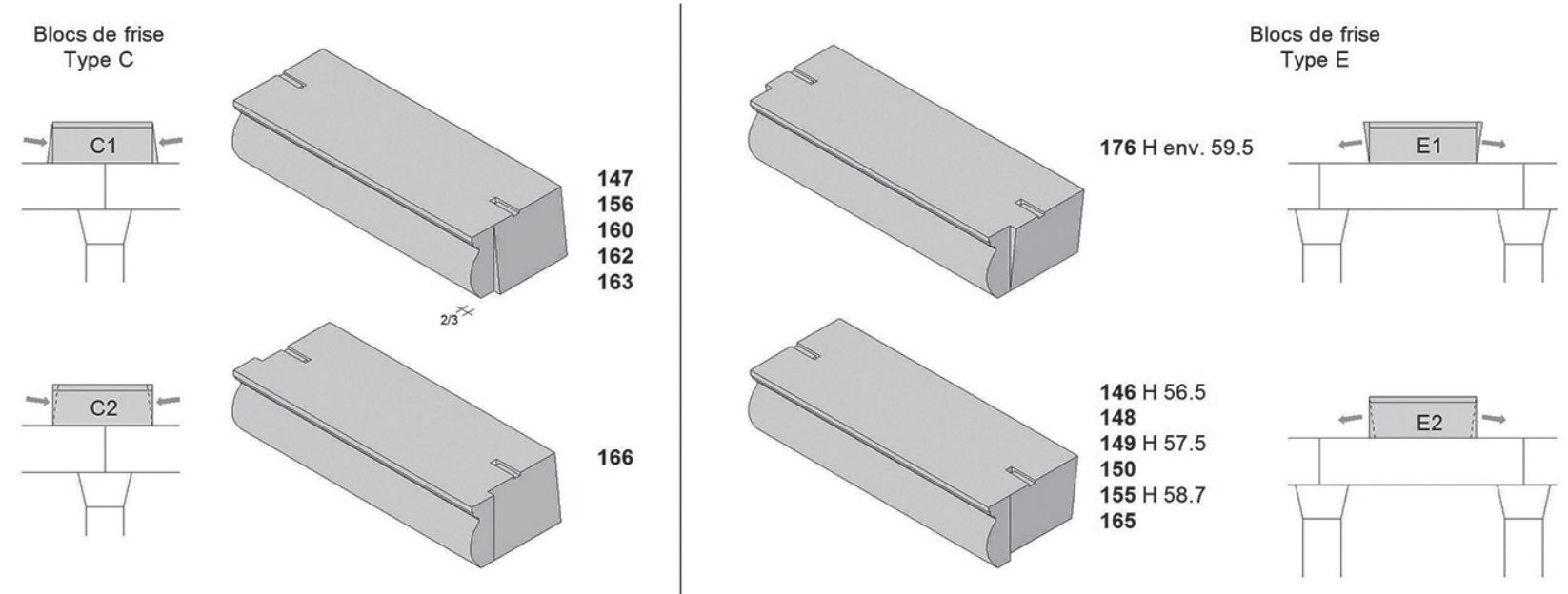

Fig. 25 : Typologie des blocs de frise en fonction de leur emplacement.

trouvait dans le naiskos axial. On restituera une statue ancienne (xoanon ?) ${ }^{22}$ dans ce dernier et une statue plus récente sur la banquette nord. En face de cette dernière se trouve une base hellénistique, antérieure au temple, si nous admettons une seule phase de construction, à l'époque romaine, pour cet édifice.

- La frise est conçue comme une série d' "arcs plats", c'est-à-dire que la pièce se trouvant entre les colonnes ne repose pas sur l'architrave mais sur les deux blocs de frise voisins se trouvant à l'aplomb de la colonne (Fig. 25). Ce dispositif ingénieux, propre à l'époque romaine, permet de réduire la hauteur de l'entablement puisque le poids des parties hautes est reporté directement sur les colonnes.
- On avait remarqué que les blocs de corniche présentaient des rythmes de denticules très variés. Après étude d'un certain nombre de blocs, on constate que ces variations ne permettent pas de distinguer plusieurs catégories, mais résultent tout simplement de la nécessité de répartir les 5 ou 6 denticules présents sur la longueur du bloc. Ces variations étaient totalement imperceptibles depuis le bas du temple.

\subsubsection{Couverture photographique du temple}

L'objectif de cette couverture photographique, réalisée par K. Konuk grâce au drone de la mission, est de réaliser un modèle $3 \mathrm{D}$ de la ruine dans son

22) Il est intéressant de noter que les monnaies d'Eurômos illustrent Zeus à la fois sous la forme d'une statue de style 'classique', mais également comme un 'xoanon' de type archaïque. 


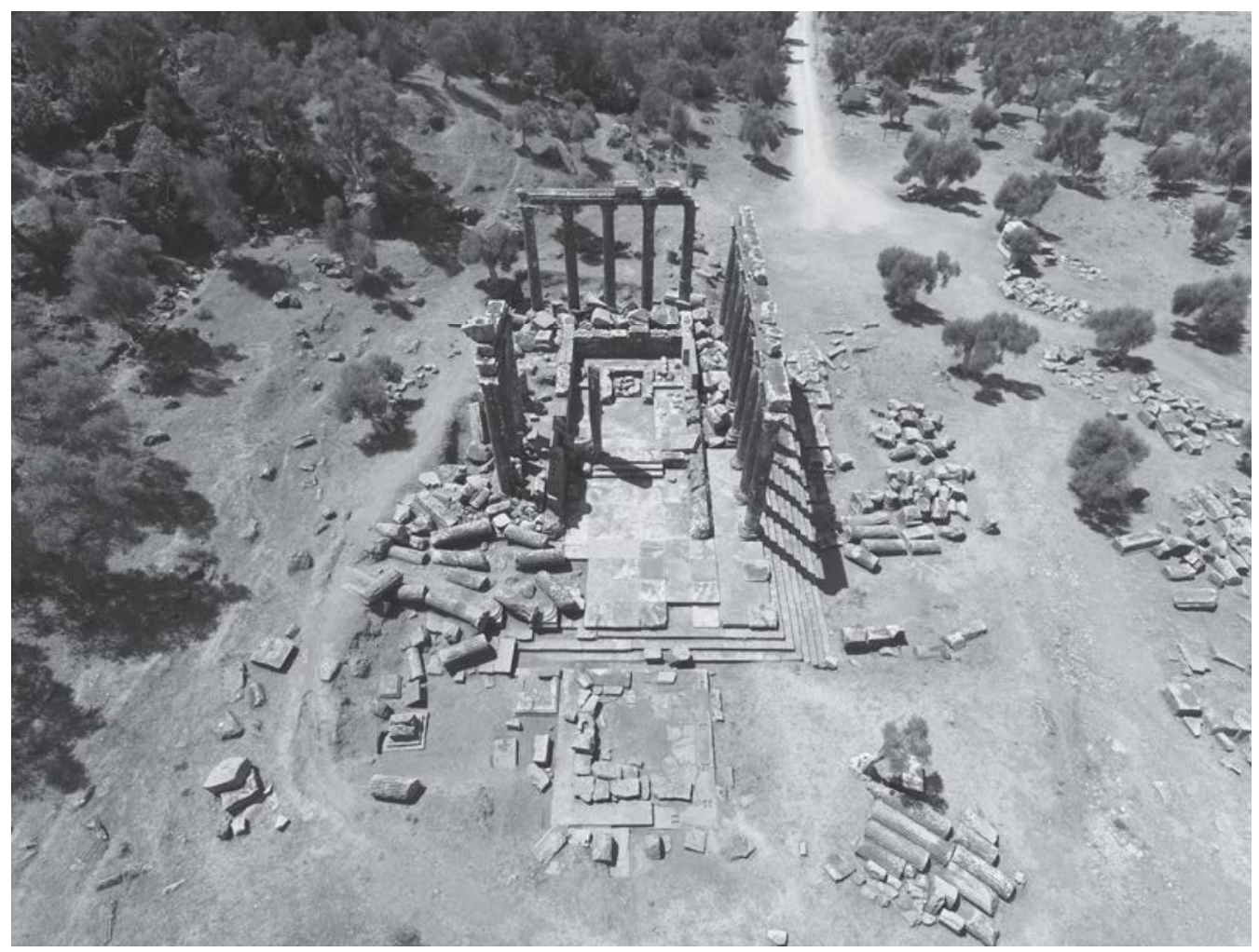

Fig. 26 : Vue aérienne du temple et de l'autel prise avec le drone.

état actuel (logiciel Photoscan). Près de sept mille photos ont été prises, couvrant l'ensemble des vestiges visibles, temple et autel, plus les alentours (notamment les dépôts de blocs) (Fig. 26). Leur traitement, en raison de la lourdeur des fichiers, nécessite une puissance de calcul dont nos ordinateurs personnels ne disposent pas. Cette modélisation se fera sur les ordinateurs du laboratoire informatique de l'université de Strasbourg, spécialisé dans ce type de travail.

\subsubsection{Travaux divers}

L'autel situé devant la façade du temple est réputé hellénistique. Il présente de grosses mortaises en queue d'aronde qui ne peuvent, à cause de leur taille, qu'avoir été préparé pour des scellements en bois. En soulevant une dalle restée en place, nous avons pu vérifier que les mortaises, vides, ne comportaient aucune trace de métal ou de corrosion (Fig. 27). Le bois, disparu depuis longtemps, assurait surtout la cohésion de la construction en phase de montage des blocs. Cette technique, par sa forme et le matériau, rappelle les techniques courantes utilisées sur les édifices égyptiens de cette époque, rapprochement que l'on peut constater à propos d'autres particularités de construction dans des monuments cariens.
Nous avons pu, en dehors des plaques d'oves qui étaient déjà connues, identifier plusieurs blocs provenant de l'autel. Ceux-ci confirment une datation

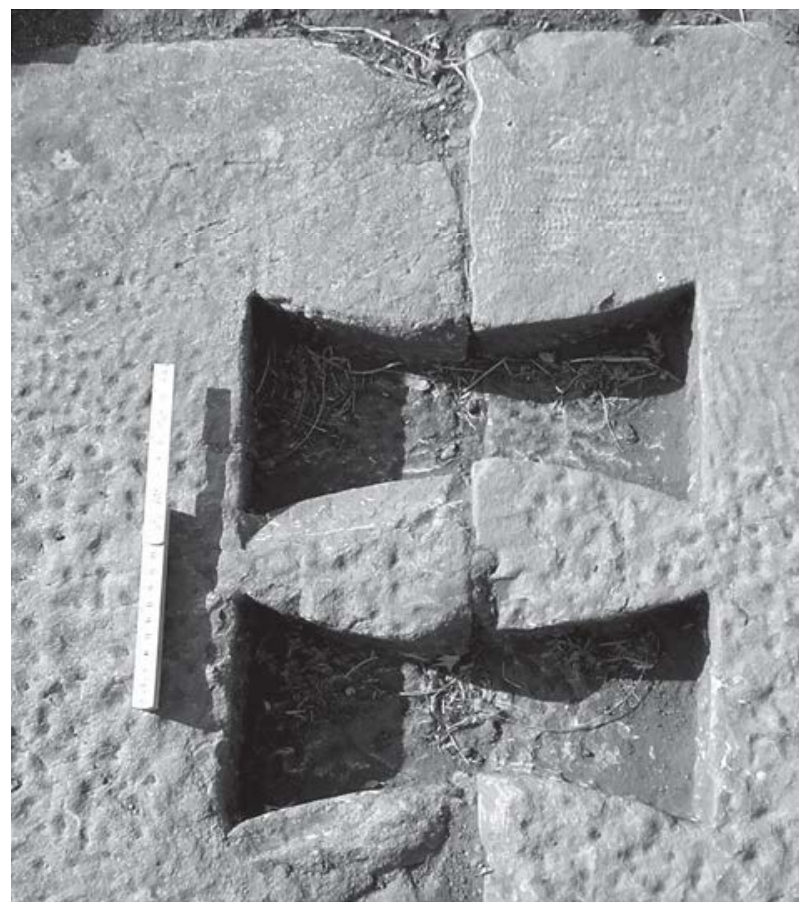

Fig. 27 : Mortaises de l'autel. 

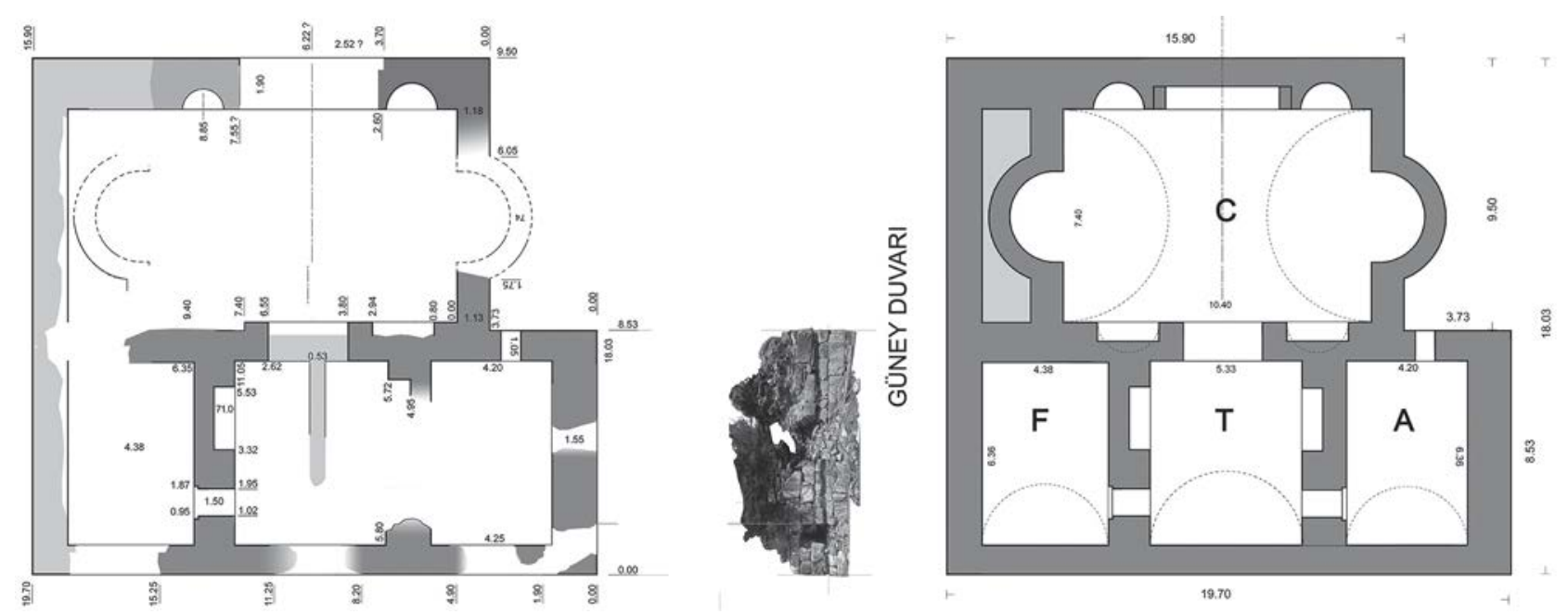

Fig. 28 : Thermes, état actuel et essai de restitution.

à l'époque hellénistique et montrent l'intérêt à inclure cette construction dans l'étude générale du temple.

\subsubsection{Perspectives de travail pour 2017}

Le catalogage des blocs doit être poursuivi et, si possible, achevé en 2017, ce qui permettra une réorganisation des dépôts de blocs. Cette réorganisation doit être pensée en fonction de l'aménagement d'aires de travail en prévision des futures interventions sur le temple (dérestauration des travaux de Ü. Serdaroğlu et restauration des parties fragilisées).

Ce travail ne peut se faire qu'avec l'aide d'un engin mécanique permettant de déplacer les blocs. En effet, la plupart des blocs sont entassés et ne peuvent êtres mesurés, photographiés et étudiés.

A condition de disposer du matériel adapté, nous procèderons à l'enlèvement des tiges de fer placées dans les blocs du temple lors des restaurations effectuées par Ü. Serdaroğlu.

Tous ces travaux préparatoires devraient permettre de présenter en 2018 un projet d'intervention sur le temple, dans le respect de l'intégrité du monument et de l'exactitude des restaurations proposées.

\subsection{THERMES DE L'AGORA}

En dehors du travail sur le temple, nous avons participé la première semaine à l'élaboration d'un dossier relatif aux thermes se trouvant au Nord de l'agora, dans la perspective du financement des restaurations nécessaires (Fig. 28).

Ces thermes, de taille modeste, étaient situés au voisinage de l'agora, comme cela est fréquent. N'étant pas encore fouillés, leur interprétation reste aléatoire. Néanmoins, on peut reconnaître, en se fondant sur la publication de F. Yegül 2006, la séquence classique Apodyterium - Tepidarium - Caldarium - Frigidarium. L'identification du Caldarium à l'Est est sûre, la répartition des trois autres espaces pourra être assurée à l'issue de la fouille quand l'accès principal du bâtiment et la présence des circuits de chauffe seront reconnus. La fouille de cet établissement nécessite la consolidation préalable des murs attaqués par des racines, notamment sur le mur sud, conservé assez haut.

\section{PROSPECTION DANS L'EURÔMIDE ET LE TERRITOIRE DE PIDASA}

L'année 2016 a vu la mise en place d'un projet de prospection accepté par la Direction des Biens culturels et des Musées et placé sous la direction d'Abuzer Kızıl. Il se propose d'étudier le territoire de la cité d'Eurômos et ses frontières avec les cités voisines. La première mission s'est donc tenue du 26.09 au 6.10 sous la supervision de Tuba Konuk, représentante du Ministère et archéologue au musée de Bilecik, dont la bienveillance a grandement facilité notre travail et à qui nous adressons nos plus vifs remerciements. Son objectif était d'étudier la partie nord-ouest de l'Eurômide, au niveau du seuil qui la sépare de la région du lac de Bafa et du territoire de la cité de Pidasa, dont nous avons publié récemment le premier décret connu.

\subsection{La région du seuil du lac de Bafa et le Nord-Ouest de l'Eurômide}

Les prospections ont débuté par une exploration de la partie nord-ouest du massif de l'Aladağ1 par l'étroite vallée nommée Kazanderesi qui part du 
village de Pınarcık. En contrebas d'un site minier de zultanite actuellement exploité nous avons visité un site antique inédit appelé Kirse Mevkii à $550 \mathrm{~m}$. d'alt. Des traces évidentes d'occupation par une petite communauté, au moins à l'époque romaine et byzantine sont visibles, ne serait-ce que par la présence de céramique commune sur une surface importante. Outre une église byzantine à double abside, il a été possible de repérer un pressoir associé à un système de presse à contrepoids (Fig. 29), ainsi qu'un dispositif de stockage enterré (pithos). Associé à ces vestiges, on note l'existence d'un mur conservé sur une longueur d'une vingtaine de mètres et une hauteur d'environ 0,90 mètre. A peu près dans l'axe du contrepoids $(90 \times 120 \mathrm{~cm})$, le mur présente une petite niche dans laquelle devait se loger la poutre (prelum) du dispositif du pressoir (Fig. 30). Toujours au même endroit, signalons l'existence d'un réservoir de stockage enterré et formé de petites pierres scellées en surface par un enduit à la chaux et dont la forme générale rappelle celle d'un pithos. Nous serions donc en présence d'un atelier de fabrication d'huile d'olive. A quelques dizaines de mètres, la présence de plaques de marbre semble indiquer le remploi de pierres tombales.

Nous avons ensuite exploré la région du seuil entre la plaine d'Eurômos et celle du golfe Latmique, au Nord du tunnel routier moderne. La découverte la plus importante a été celle d'une petite forteresse byzantine (Fig. 31) à $250 \mathrm{~m}$ d'alt. au sommet du Kale Tepe qui complète le dispositif byzantin de défense de la région et de la route étudié autrefois par Th. Wiegand et W. Müller-Wiener. Immédiatement au Nord-Ouest, à une centaine de mètres à vol d'oiseau, dans le petit bassin de Dikenlik Sirtı Mevkii existe un modeste site antique bâti au sommet d'un petit éperon dont les traces remontent au moins à l'époque romaine.

Dans la partie nord de l'Eurômide, le travail de prospection s'est ensuite centré autour du village d'Ekindere. A l'extérieur du village actuel, à trois cents mètres vers l'Ouest, une importante nécropole carienne est repérable, au tout début de la pente. Une trentaine de tombes ont été creusées dans la roche. Toutes sont évidées et l'on note l'absence des plaques supérieures, évidemment remployées. Le parcours du terroir du village au Sud dans la plaine, entre autres sur le site appelé Ören ou Karacaöz Çayı Mevkii, permet de repérer de nombreuses traces d'occupation et d'exploitation agricole antiques (blocs, tuiles, maie, fragments de colonnettes).

\subsection{Pidasa : sites, terroir et territoire}

La communauté antique de Pidasa occupe un site singulier en raison de son étagement extrême sur la face orientale du massif du Grion (aujourd'hui Aladağ 1 ). Nous avons pu faire une première étude d'ensemble des différents secteurs situés dans les montagnes et sur les plateaux qui dominent les villages actuels de Kurudere et Eğridere et, plus largement, la plaine de Selimiye.

\subsubsection{Le site de Pitese}

A mi-distance entre les villages de Kurudere et d'Eğridere, dans la plaine mais au pied de la pente, en contrebas du plateau de Bozseki Mevkii, se situe le lieu-dit Pitese, également appelée Düdüklü Mevkii. Sur un espace assez étendu, aujourd'hui occupé par les oliveraies et les activités d'élevage, il est possible de repérer de nombreuses tombes antiques dispersées, souvent d'aspect assez tardif. Une inscription funéraire d'époque byzantine a été relevée en association avec ces structures funéraires.

\subsubsection{Le plateau de Bozseki (vers $240-260 \mathrm{~m}$ )}

A partir du village de Kurudere, il est possible de suivre un chemin qui mène, au terme d'une vingtaine de minutes de marche, à un plateau arboré dominant le village (Bozseki Mevkii, Fig. 32). Ce plateau, situé à une altitude de 240-260 m, est occupé par des oliveraies et utilisé comme lieu de pacage et marqué par la présence de nombreux murets modernes liés à cette activité. C'est aussi un site antique et plusieurs éléments et structures sont repérables.

Sur le rebord du plateau, orientés vers l'Est et le Nord-Ouest, c'est-à-dire face à la plaine, un mur de terrasse constitué partiellement de blocs taillés est clairement visible et peut être suivi sur $27 \mathrm{~m}$ ainsi que le début des murs de retour prolongés par des assises (sur $50 \mathrm{~m}$ sur le côté oriental) (Fig. 33). A partir des retours, et en dépit de la disparition de toute élévation sur le plateau, on repère un important bâtiment quadrangulaire qu'il est possible d'identifier comme le sanctuaire de Toubassis connu par l'inscription que nous avons publiée ${ }^{23}$. Sur l'assise de ce qui était l'entrée du bâtiment, un seuil et des traces de portes sont visibles, ainsi que des traces de colonnes. Cette assise était redoublée à l'intérieur du bâtiment, sans présence apparente de colonne, de façon à définir deux espaces séparés, de superficies inégales. A proximité de cet ensemble, on repère 


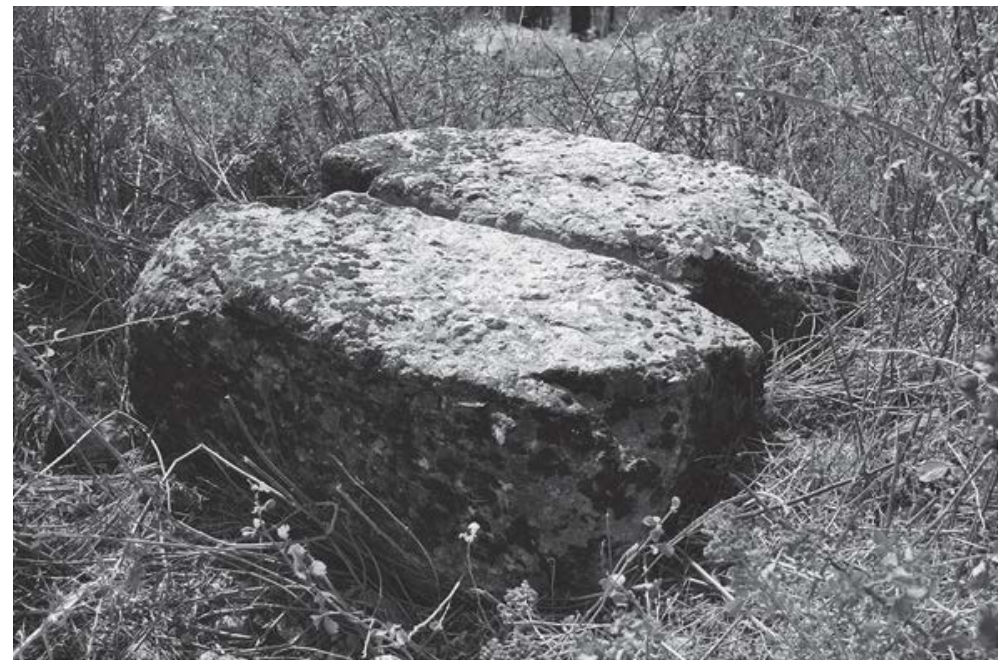

Fig. 29 : Contrepoids en pierre calcaire appartenant à un dispositif de presse.

Fig. 30 : Mur présentant une niche destinée vraisemblablement à recevoir la poutre d'un dispositif de presse.
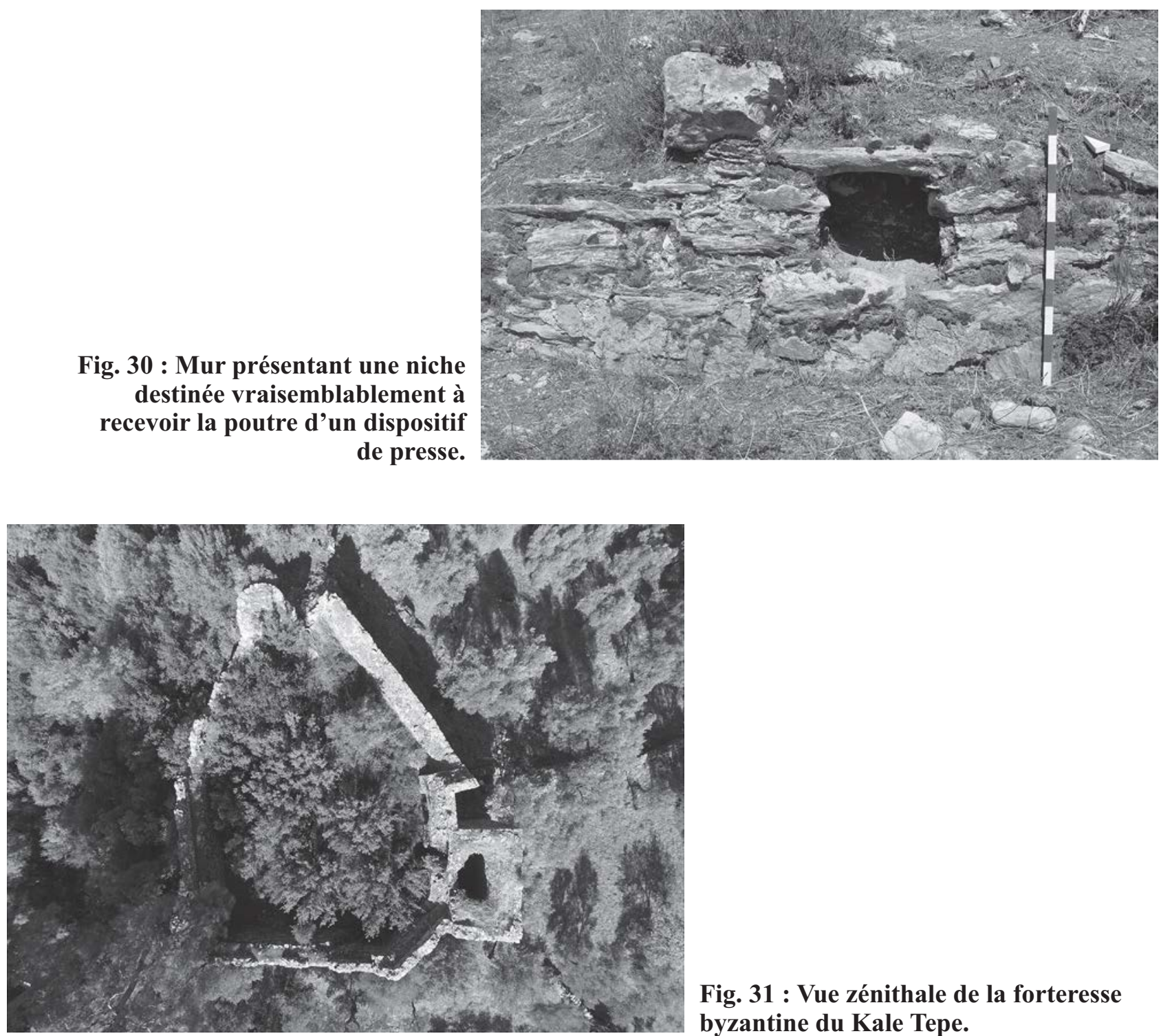

Fig. 31 : Vue zénithale de la forteresse byzantine du Kale Tepe. 


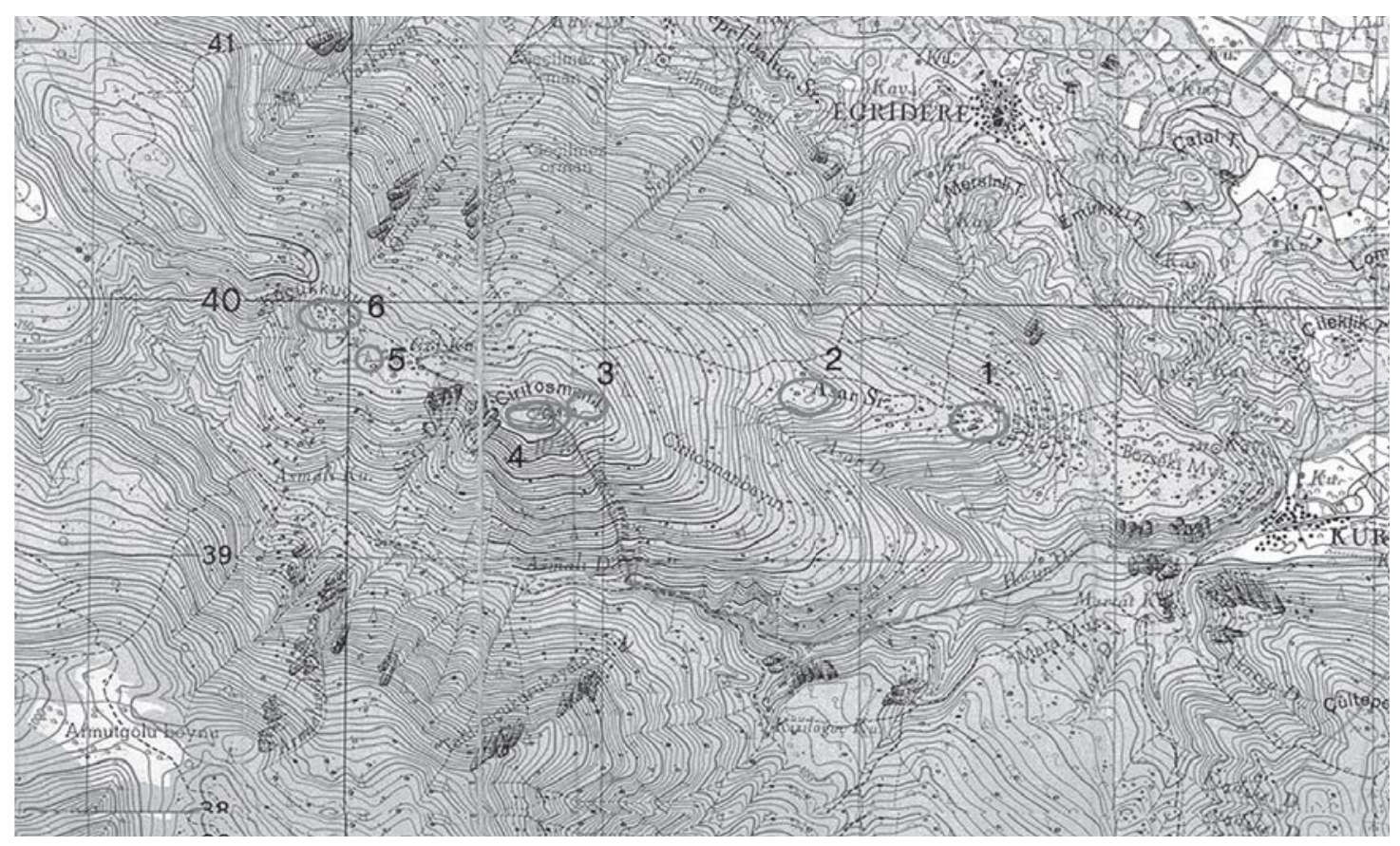

Fig. 32 : Carte indiquant les différents sites étudiés dans le territoire de Pidasa.

également des structures de fortification avec tour ainsi que plusieurs blocs isolés et un tambour de colonne.

Immédiatement en contrebas, à l'Est de la terrasse du bâtiment principal, une fouille clandestine récente a révélé la présence d'un tombeau datable de la fin $\mathrm{du} 4^{\mathrm{e}}$ s. av. J.-C. ${ }^{24}$ fermé par une porte. Construit pour 6 ou 7 personnes déposées chacune sur des lits superposés, sur trois niveaux.

L'exploration plus attentive du plateau a permis de prendre conscience de l'importance de ce site. On note d'abord dans la partie ouest-sud-ouest un bâtiment byzantin qui pourrait être une église, car un des angles montre un net arrondi de type absidial (Fig. 34). Un peu plus loin, à une distance approximative de $150 \mathrm{~m}$ de la terrasse, on trouve beaucoup de blocs de grande dimension (de 1,50 $\mathrm{m}$ à plus de $2 \mathrm{~m}$ ) pouvant servir de linteaux ou de montants de porte (Fig. 35) et, surtout, un chapiteau d'ante éolique (Fig. 36) datable de la seconde moitié du $6^{e} \mathrm{~s}$. av. J.-C., semblable à ceux d'Alazeytin/Telmessos, de Pedasa ou d'Eurômos.

A côté de ces éléments, dans le même secteur, on relève la présence d'un fragment de coupe à vernis noir qui pourrait dater du $4^{\mathrm{e}} \mathrm{s}$. av. J.-C., de beaucoup de blocs de très grande taille, dont certains font penser à des stèles de même forme dont nous ignorons pour l'instant la fonction. Deux d'entre elles disposent de décorations moulurées en forme de palmette dont le style indiquerait la fin de la période archaïque (Fig. 37). Signalons également la présence de maies à huile en quantité, parfois très petites comme s'il s'agissait de lieux de libations. Tout semble indiquer la présence de tombes et d'un habitat important, centre peut-être de la cité de Pidasa.

\subsubsection{Asar intermédiaire (vers $410-450 \mathrm{~m}$ )}

Le second niveau d'occupation du site de Pidasa se situe sur un premier épaulement, à un peu plus de $400 \mathrm{~m}$ d'altitude (Fig. 32: 1). Sur le rebord oriental, on relève la présence d'une imposante muraille de plan trapézoïdal en pierres sèches qui, au prix d'un décrochement, s'engage résolument dans la pente du côté sud, sur une centaine de mètres. La fortification semble avoir eu une circonférence de $450 \mathrm{~m}$ environ (Fig. 38). Une tour est visible sur la partie nord. L'appareil polygonal est parfaitement caractéristique des murailles dites "cariennes", ou encore "lélèges",

24) Une monnaie de bronze de Philippe III Arrhidée frappée à Milet a échappé à l'attention des fouilleurs clandestins et a été récupérée lors de la fouille de sauvetage organisée par le musée archéologique de Milas. De la céramique de la même époque a pu être également collectée par les archéologues. 


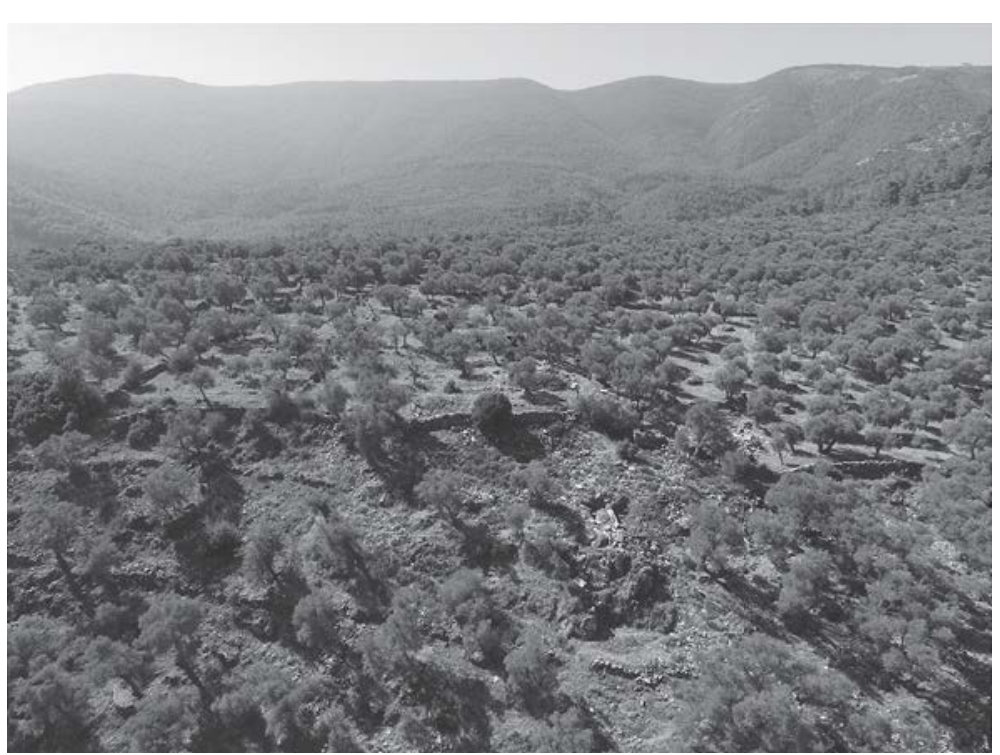

Fig. 33 : Vue aérienne du plateau de Bozseki prise depuis le rebord nord-est.

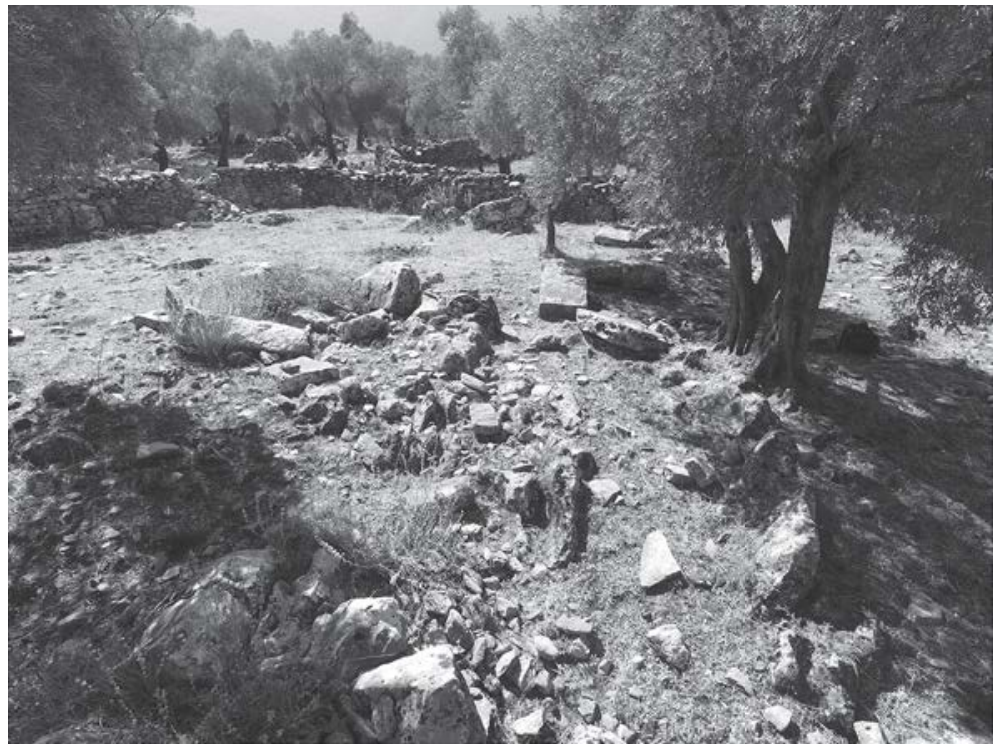

Fig. 35 : Secteur présentant une concentration importante de blocs d'architecture.

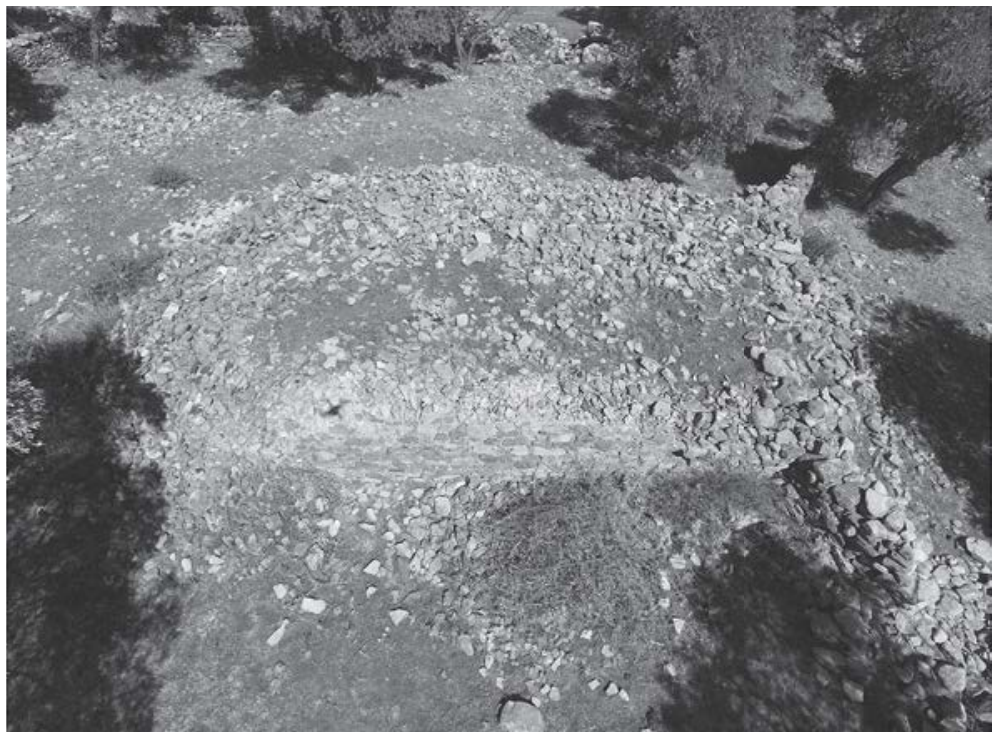

Fig. 34 : Bâtiment qui pourrait être une petite église byzantine.

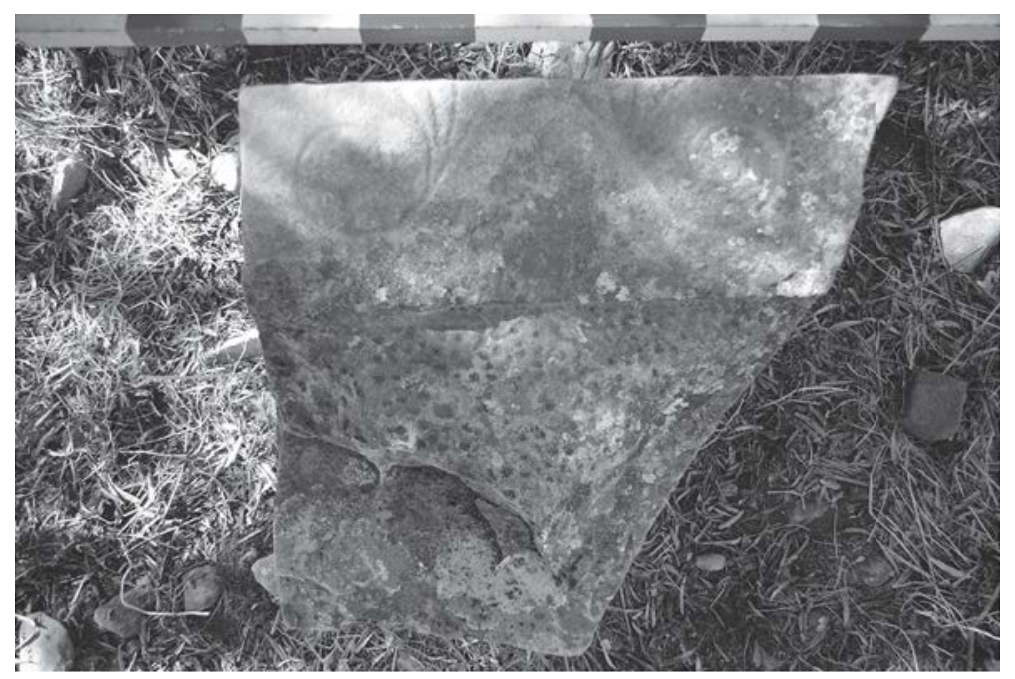

Fig. 36 : Chapiteau d'ante éolique. 


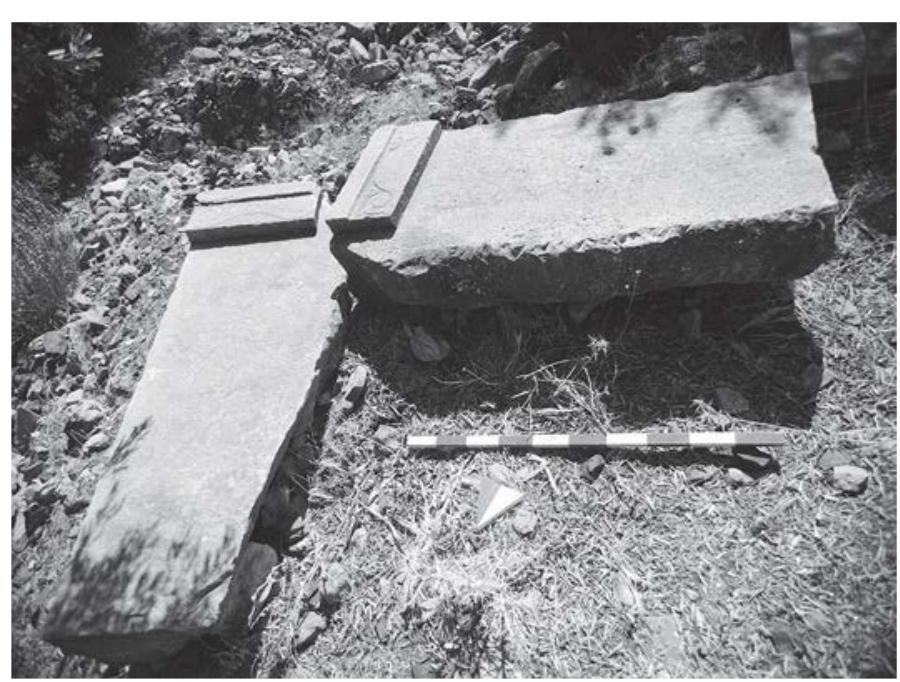

Fig. 37 : Deux stèles décorées de palmettes.

nombreuses dans la région. La structure est largement effondrée sur elle-même. Quelques traces de céramiques ont pu être repérées dans la pente orientée au Sud-Est du kale. Le centre de cette fortification est couverte de végétation et ne présente pas de structure évidente.

En prolongeant le parcours plus à l'Ouest, sur 400 ou $500 \mathrm{~m}$, on peut accéder à un second épaulement (520 m. d'alt.), l'Asar proprement dit. On y relève un second système de défense (Fig. 32: 2), assez complexe, mais sans doute plus modeste dans son emprise que la forteresse située juste en dessous. Au moins deux murs en pierres sèches sont repérables vers le Nord. Le Sud est protégé par le rocher naturel. Dans la partie supérieure, la fortification a été reprise, probablement à l'époque hellénistique: on relève la construction d'une tour dans un appareil frustre mais régulier (dimensions : environ $16 \mathrm{~m}$ sur $6 \mathrm{~m}$ ) et deux salles distinctes sont repérables à l'intérieur.

\subsubsection{L'occupation sommitale : le Cerit Osman Kalesi}

La partie la plus élevée est elle-même composés de plusieurs sous-ensembles. Sur un épaulement orienté vers l'Est, en contrebas immédiat du sommet, il a été possible de repérer plusieurs bâtiments, notamment une structure massive associée à une voûte en pierre, effondrée, dont on peut voir clairement le départ (Fig. 32: 3). Juste au-dessus, un grand bâtiment orienté vers le Sud-Est présente de belles assises régulières qui sont conservées en totalité, décorées et surmontées par une seconde rangée de blocs d'assise, plus grossièrement taillés, puis par des orthostates

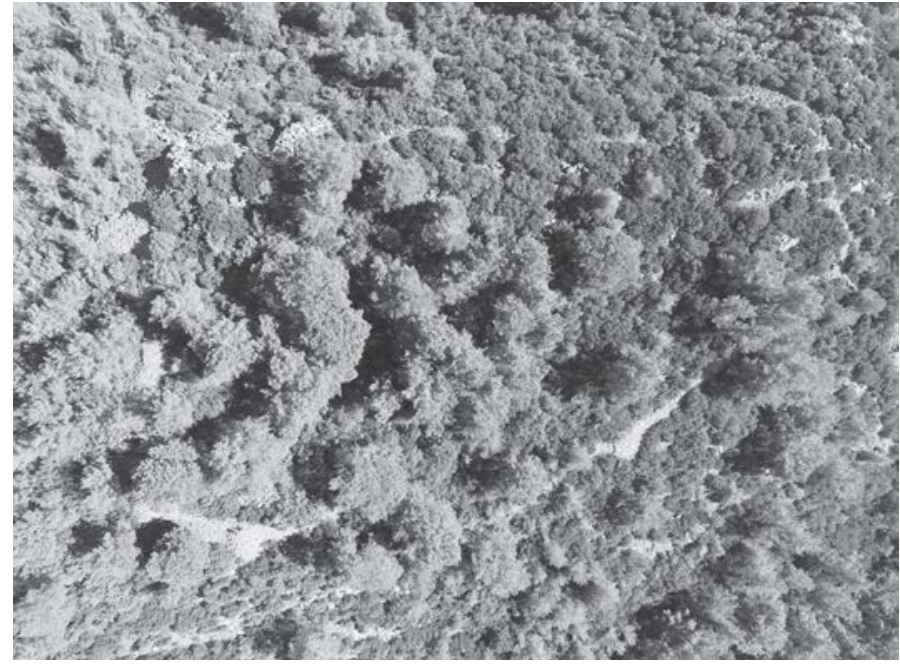

Fig. 38 : Vue zénithale du site fortifié de l’Asar Tepe.

dont beaucoup sont visibles (Fig. 39). On relève aussi la présence de puissants linteaux mais pas de traces de colonnes ou de tuiles. L'ensemble a été récemment pillé. L'espace central est divisé en deux parties inégales (dimensions totales de 14,5 m/10,8 $\mathrm{m}$ avec une salle principale : 10,8 m/6,6 m. Epaisseur du mur intérieur : $1,5 \mathrm{~m}$ ). Une datation à l'époque hécatomnide ou au début de l'époque hellénistique semble possible. L'hypothèse qu'il puisse s'agir d'un temple ou d'un hérôon n'est pas à exclure, tant l'aspect du bâtiment et le soin apporté à sa réalisation sont impressionnants.

Au sommet oriental du Cerit Osman Kalesi (830 m) se trouve ce qui semble avoir été la forteresse principale, qui épouse fidèlement un long éperon ellipsoïdal (Fig. 32: 4 et 40). Composée de deux lignes de fortification, au moins dans sa partie orientale, elle présente les traces de deux tours sur le rebord oriental de l'éperon rocheux et de plusieurs autres tours, orientées vers le Nord. Les traces d'une occupation intérieure sont très nombreuses (murs de bâtiments, présence de céramique).

En quittant cette forteresse, on s'engage vers l'Ouest dans la longue dépression sommitale. Une très belle tour hécatomnide ou hellénistique, signalée rapidement par W. Radt a été étudiée (Fig. 32: 5). On relève une belle élévation de plus de 3 mètres, avec une composition en carreaux et boutisses et la présence de bossage (Fig. 41). Il est encore possible d'identifier deux salles et une porte intérieure (dimensions : hauteur 3,6 m; largeur 9,4 m; longueur: $12,5 \mathrm{~m}$; largeur des murs $1,2 \mathrm{~m}$ ).

Enfin, le sommet de Pidasa est occupé par une dernière forteresse (Fig. 32: 6), située sur l'éperon occidental $(800 \mathrm{~m})$. Plus modeste que la structure 


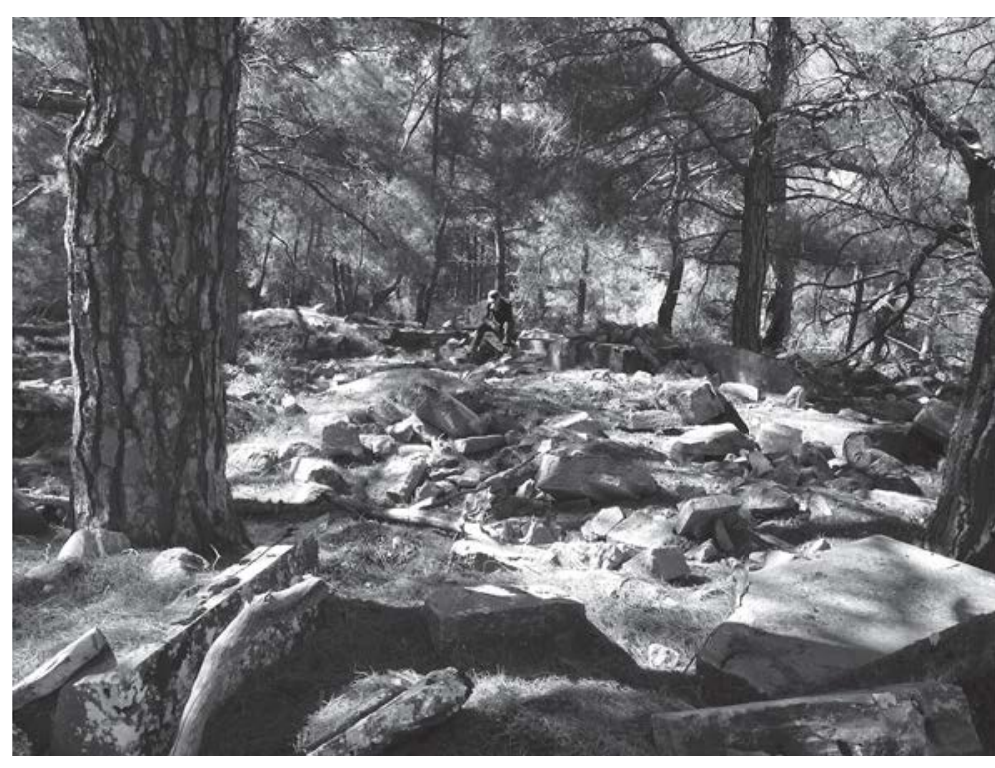

Fig. 39 : Ruines du bâtiment en contrebas de la forteresse de Cerit Osman Kalesi.

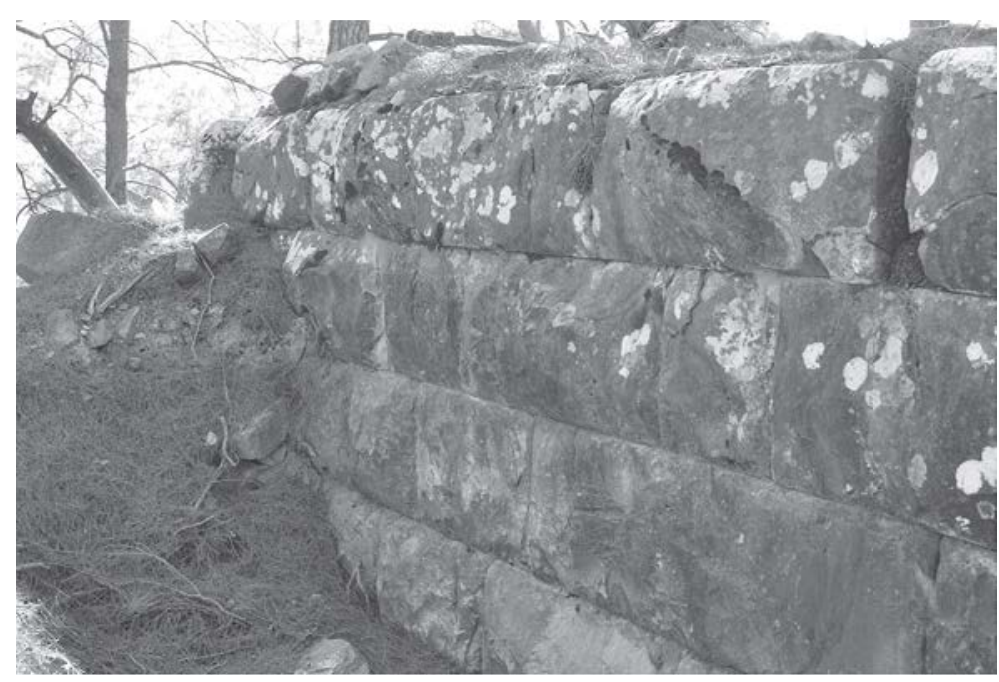

Fig. 41 : Mur est de la tour en contrebas de Cerit Osman Kalesi.

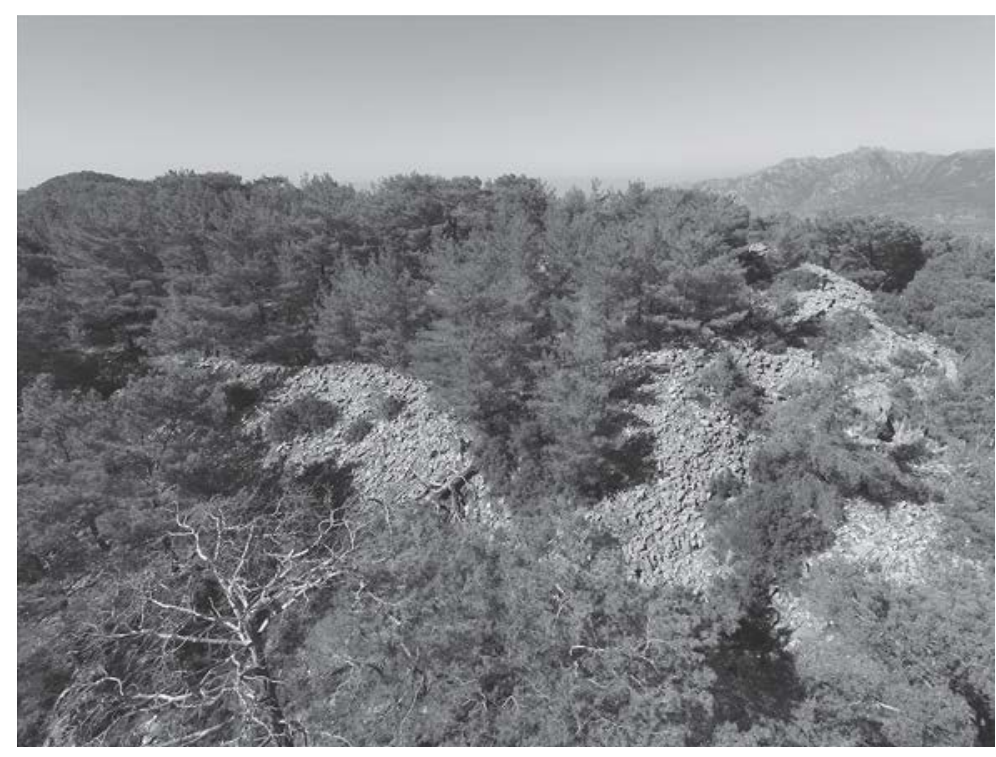

Fig. 40 : Vue aérienne prise depuis le Sud de la forteresse de Cerit Osman Kalesi.

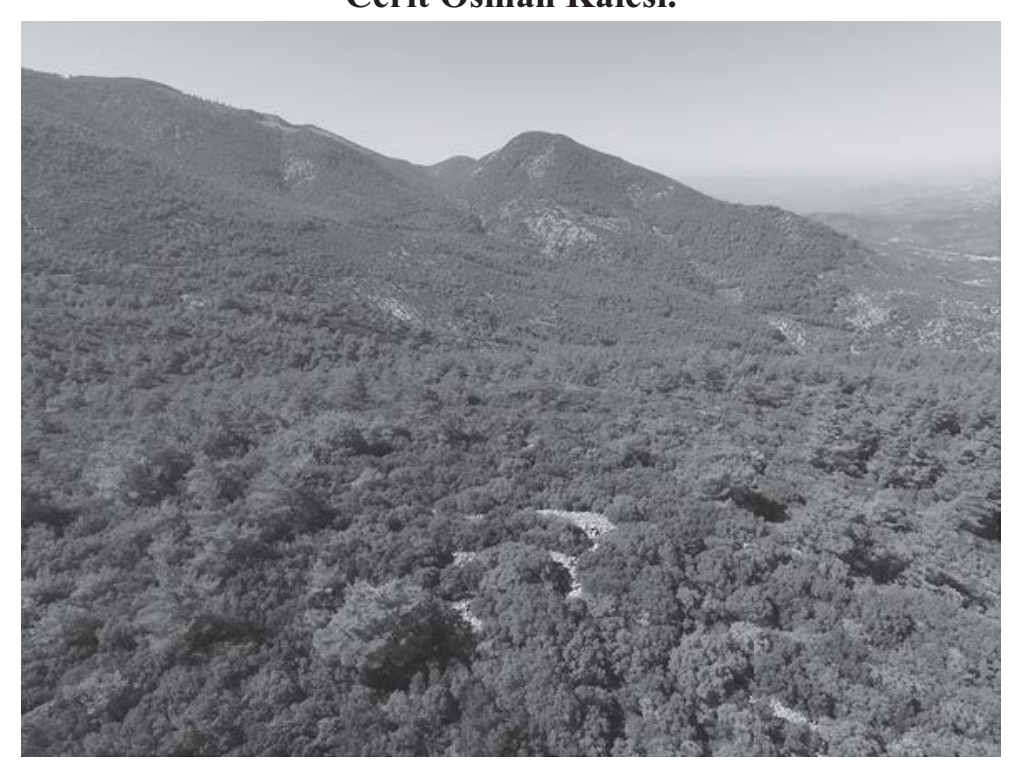

Fig. 42 : Vue aérienne prise depuis le Sud de la petite forteresse de Nasuf(un) Gölü, à l'arrière plan : le sommet de Cerit Osman Kalesi. 
orientale, elle présente aussi des murs en polygonal irrégulier. Au moins deux lignes de murs sont repérables et de nombreux alignements sont en place. On relève la présence d'une tour centrale mais les traces d'occupation intérieure sont beaucoup plus faibles que dans la forteresse orientale. En contrebas de cette éminence, sur le versant nord-est, on note la présence de nombreux blocs imposants appartenant sans doute à une nécropole antique.

Nous avons enfin commencé à étudier le territoire au Sud de Pidasa et qui forme la frontière occidentale de la cité d'Eurômos. Immédiatement au Sud du village de Kurudere dans une région très difficile d'accès du fait de la végétation, nous avons découvert vers 410 m. d'alt., au lieu-dit Nasuf (ou Nasufun) Gölü, une petite forteresse qui gardait en rapport avec l'ensemble de Pidasa la route vers Milet (Fig. 42). La technique de construction est en polygonal irrégulier de type appelé par commodité "lélège". On notera cependant que le plan, les tours carrées et le double parement invitent à ne pas exclure une construction à l'époque hellénistique.

En résumé, le travail de prospection de cette année a été centré sur l'occupation de l'espace et le contrôle des territoires, notamment sous l'angle des systèmes défensifs. Il a permis aussi d'avancer dans la compréhension de l'organisation du site de Pidasa, à la frontière nord de l'Eurômide, un site impressionnant et singulier par son étagement, mais aussi par l'importance de l'occupation sommitale comme par la polarisation autour du sanctuaire de Toubassis dès l'époque archaïque. L'importance des résultats de cette première année permet d'envisager une étude plus complète de la structure d'ensemble de cette partie de l'Eurômide autour du remarquable site de Pidasa, jusqu'à présent très mal compris, et de tout le système de défense occidental de la plaine de l'Eurômide.

A.K. et al.

\section{BIBLIOGRAPHIE} Paris.

Adam, J.-P., 1982 : L'architecture militaire grecque,

Brun, P., 1994 : "Les fortifications d'Hyllarima, Philon de Byzance et Pleistarchos", REA 96 : 193-204.

Debord, P. et Varinlioğlu, E., 2011 : Cités de Carie, Harpasa, Bargasa, Orthosia dans l'Antiquité, PUR, Rennes.

Hellmann, M.-C., 2010 : L'architecture grecque 3. Habitat et fortifications, Picard, Paris.

Kienast, H.J., 1978 : Samos XV. Die Stadtmauer von Samos, Bonn.

Kızıl, A., Brun, P., Capdetrey, L., Descat, R., Fröhlich, P. et Konuk, K., 2015 : "Pidasa et Asandros : une nouvelle inscription (321/0)", REA 117/2:371-409.

Kızıl, A., Konuk, K., Brun, P., Capdetrey, L., Descat, R., Fröhlich, P., Laroche, D., Le Quéré, E., Prost, F. et Vergnaud, B., 2016 : "Eurômos : rapport préliminaire sur les travaux réalisés en 2015", Anatolia Antiqua XXIII (2016) : 321-338.

Martini, W., 2016 : "Form, Funktion und Bedeutung der Stadtmauern von Perge in Pamphylien", in Frederiksen, R., Müth, S., Schneider, P. et Schnelle, M. (éds.), Focus on Fortifications. New Research on Fortifications in The Ancient Mediterranean and The Near East. Acropolis Museum in Athens, 6-9 December 2012. (Fokus Fortifikation Studies. vol. 2), Oxbow Books, Oxford : 220-231.

McAllister, M.H., 2005 : The Excavations at Ancient Halieis: The Fortifications and Adjacent Structures (Vol. 1), Indiana University Press, Bloomington.
McNicoll, A.W., 1997 : Hellenistic fortifications from the Aegean to the Euphrates, Oxford.

Pimouguet-Pédarros, I., 2000 : Archéologie de la défense : Histoire des fortifications antiques de Carie aux époques classique et hellénistique, Presses Universitaires de Franche Comté.

Vergnaud, B., 2014 : "Quelques observations sur la forteresse de Labraunda", in Karlsson, K., Carlsson, S. et Blid Kullberg, J. (éds.), LABRYS. Studies presented to Pontus Hellström, Uppsala University (BOREAS. Uppsala Studies in Ancient Mediterranean and Near Eastern Civilizations 35) : 107-122.

- 2015: "La forteresse de l'acropole de Labraunda. Rapport préliminaire sur la $3^{\mathrm{e}}$ campagne de fouille (2014)", in Henry, O. et al., "Labraunda 2014", Anatolia Antiqua XXIII : 316-330.

- 2016a : "La forteresse de l'acropole de Labraunda. Rapport préliminaire sur la 4 e campagne de fouille (2015)", in Henry, O. et al, "Labraunda 2015", Anatolia Antiqua XXIV : 397-412.

- 2016b : "La muraille d'Eurômos 2014-2015", in Kızıl, A. et al., "Eurômos : Rapport préliminaire sur les travaux réalisés en 2015", Anatolia Antiqua XXIV : 325333.

Von Gerkan, A., 1935 : Die Stadtmauern, Milet II. 3, Berlin.

Winter, F.E., 1971 : Greek fortifications, Toronto.

Yegül, F., 2006 : Antik Çăg'da Hamamlar ve Yıkanma, Istanbul. 